\title{
A UNIFYING PICTURE OF GENERALIZED THERMODYNAMIC UNCERTAINTY RELATIONS
}

\author{
A.C. BARATO, R. CHETRITE, A. FAgGionAtO, AND D. GABRIELLI
}

\begin{abstract}
The thermodynamic uncertainty relation is a universal trade-off relation connecting the precision of a current with the average dissipation at large times. For continuous time Markov chains (also called Markov jump processes) this relation is valid in the time-homogeneous case, while it fails in the timeperiodic case. The latter is relevant for the study of several small thermodynamic systems. We consider here a time-periodic Markov chain with continuous time and a broad class of functionals of stochastic trajectories, which are general linear combinations of the empirical flow and the empirical density. Inspired by the analysis done in our previous work [1, we provide general methods to get local quadratic bounds for large deviations, which lead to universal lower bounds on the ratio of the diffusion coefficient to the squared average value in terms of suitable universal rates, independent of the empirical functional. These bounds are called "generalized thermodynamic uncertainty relations" (GTUR's), being generalized versions of the thermodynamic uncertainty relation to the time-periodic case and to functionals which are more general than currents. Previously, GTUR's in the time-periodic case have been obtained in 1, 27, 42. Here we recover the GTUR's in 1, 27. and produce new ones, leading to even stronger bounds and also to new trade-off relations for time-homogeneous systems. Moreover, we generalize to arbitrary protocols the GTUR obtained in [42] for time-symmetric protocols. We also generalize to the time-periodic case the GTUR obtained in [19 for the so called dynamical activity, and provide a new GTUR which, in the time-homogeneous case, is stronger than the one in [19]. The unifying picture is completed with a comprehensive comparison between the different GTUR's.
\end{abstract}

\section{INTRODUCTION}

The thermodynamic uncertainty relation (TUR) recently introduced in [3] is a universal inequality that relates the precision of any current, such as the velocity of a molecular motor or the electron flux in a quantum dot, with the entropy production that quantifies energy dissipation. More precisely, the ratio of the asymptotic diffusion coefficient of any current to its squared asymptotic value is lower bounded by the inverse average entropy production rate. This relation constitutes a key result in stochastic thermodynamics [44, 45], a theoretical framework that extends thermodynamics to small nonequilibrium systems. More generally, the TUR is a consequence of a parabolic bound on large deviations (LD) proposed in [21, 35]. The proof of this bound, which has been obtained in [21], comes from the explicit form derived in [6, 7, 30] of the rate functional associated with the so called 2.5 level LDs.

Several works about the TUR and quadratic bounds on LD rate functionals have already been produced (see for example [1, 2, 8, 9, 10, 11, 12, 13, 16, 19, 20, 23,

This work has been supported by Italian PRIN 20155PAWZB "Large Scale Random Structures" and by the project "Investissements d'Avenir" UCA JEDI of the French ANR n. ANR-15-IDEX-01. 
24, 26, 27, 28, 29, 32, 33, 34, 36, 37, 38, 39, 40, 41, 42, and references therein). In particular, the TUR applies to systems driven by a fixed thermodynamic force. Mathematically, these systems can be described as time-homogenous Markov chains, i.e. with time-independent transition rates, or time-homogeneous diffusions as in [22, 32, 41]. A different way to drive a system out of equilibrium is through an external periodic protocol. Several artificial molecular pumps [17] and colloidal heat engines [31] constitute experimental examples of such periodically driven systems. A continuous-time Markov chain with time-periodic transitions rates is a standard mathematical framework to describe these systems [4].

As shown in [4, there is a fundamental difference between systems driven by a fixed thermodynamic force and periodically driven systems concerning the TUR. The original TUR from [3] that involves the entropy production does not apply to periodically driven systems. However, more recently, bounds on current fluctuations that generalize the TUR to periodically driven systems have been obtained in 11, 27, 42. In this work we focus on generalized thermodynamic uncertainty relations (shortly, GTUR's). In a very broad sense, given a class of empirical functionals, by GTUR we mean a lower bound on the ratio of the asymptotic diffusion coefficient to the squared asymptotic value of the empirical functional, which holds uniformly as the empirical functional varies in the given class, in the sense that the lower bounding quantity does not depend on the specific empirical functional and depends only on the Markov process itself and the class of functionals under consideration.

A summary of the GTUR's developed so far (cf. [1, 27, 42]) is as follows. A first GTUR for periodically driven systems has been provided in [42]. This result is restricted to protocols that are time-symmetric under time reversal and to the class of empirical functionals fulfilling an antisymmetry relation. The resulting lower bound is in terms of the averaged entropy production rate, although in a form different from the standard TUR. A second contribution has come from our previous work [1]. There we have presented a very general method to get local quadratic upper bounds on the LD rate function of currents, and therefore lower bounds on the ratio of the asymptotic diffusion coefficient to the squared asymptotic value. As an application, we have obtained several specific classes of lower bounds (cf. 11. Eq. (55),(56),(61),(72),(73),(74)]), which hold for generic currents, also with time-dependent increments (the increment is the variation of the current due to a transition). When restricting to time-independent increments several lower bounds provided in [1] become uniform w.r.t. the possible increments and therefore are GTUR's, in the sense specified above (cf. e.g. 11, Eq. (26),(27)]). Another GTUR has been derived in 27. for a class of empirical functionals given by a current and a generic term that is linear in the fractions of time spent in a state, the so called empirical density (or measure).

Part of our main results are an extension of the analysis performed in [1]. We consider a quite broad class of empirical functionals. This class includes currents, which are the standard observables that appear in the TUR, an observable known as activity that has symmetric increments [19] (in contrast to currents that have antisymmetric increments) and the empirical density. In fact, our GTUR's are generalizations of the TUR in two senses: we consider time-periodic Markov chains and empirical functionals more general than currents. For instance one of our GTUR's is a generalization to the time-periodic case of the bound found in [19] related to the 
dynamical activity. We remark that, even for currents and time-homogeneous processes, some of our GTUR's are different and tighter than the usual TUR (similarly, one of our GTUR's is tighter than the bound found in [19] related to the dynamical activity). Finally, these GTUR's should not be confused with the generalizations of the TUR to finite time in time-homogeneous, time-inhomogeneous or time-periodic systems obtained in [11, 12, 13, 26, 38.

We provide general methods to produce local quadratic upper bounds on the LD rate function of the empirical functionals (cf. Theorems 112 and 3). These methods rely on the LD principles obtained in [5] and work whenever one can exhibit a suitable mathematical object, that we call here legal input. By choosing suitable legal inputs we get the different GTUR's listed in Section 3 as (GTUR 1), (GTUR 2),..., (GTUR 6). In this way we recover the results of [1, 27] but also go further, exhibiting new GTUR's which are sometimes even stronger of the existing ones (for example, (GTUR 4) provides always a stronger lower bound than the GTUR in [27]).

The GTUR in 42 is of a different nature. Our unifying picture is completed with a generalization of this GTUR to the case of general protocols that can be time-asymmetric (cf. (GTUR 7) in Section 3). This GTUR applies to a class of functionals that fulfills an antisymmetry relation. Interestingly, the average entropy production rate that appears in the bound for the case of symmetric protocols is substituted by an average naive entropy production rate introduced in [5]. This rate equals the rate of entropy production plus a rate that becomes zero if the protocol is symmetric.

All our results apply as well to time-homogeneous Markov chains with continuous time, since they are a special case of periodically driven systems. In particular, our GTUR's include the original TUR from [3] and imply a generalization of the bound on the fluctuations of activity derived in [19].

Outline of the paper. In Section 2 we fix the notation, describe the model and the empirical functionals we will focus on. In Section 3 we present our main GTUR's, denoted by (GTUR 1), (GTUR 2),..., (GTUR 7). In Section 4 we discuss in detail two examples. In Sections 5 and 6 we provide general methods (cf. Theorems 1 , 2 and 3 there) to get local quadratic upper bounds on the LD rate function and derive all the GTUR's listed in Section 3, apart from (GTUR 7), as well as some other lower bounds on the ratio between speed and precision (cf. Corollaries 5.2 and 6.2). In Section 7 we extend the results of [42] to generic protocols (cf. Theorem 4), and derive (GTUR 7). Finally, we collect some general remarks and proofs in the Appendixes.

\section{Notation AND General FRAMEWORK}

2.1. Models and notation. We consider a continuous-time Markov chain $X(t)$ with finite state space $V$ and time-periodic jump rates $w_{i j}(t)$ with period $\tau$ :

$\mathbb{P}(X(t+d t)=j \mid \xi(t)=i)=w_{i j}(t) d t, \quad w_{i j}(t+\tau)=w_{i j}(t) \quad \forall i, j \in V, \forall t \geq 0$.

The transition graph associated with the Markov chain $X(t)$ is denoted $(V, E)$, with vertex set $V$ and set of oriented edges $E$. Our main technical assumptions are the following:

(i) the graph $(V, E)$ is strongly connected; 
(ii) for each $(i, j) \in E$ it holds $w_{i j}(t)>0$ for all $t$, while for each $(i, j) \notin E$ it holds $w_{i j}(t)=0$ for all $t$.

We recall that Item (i) is equivalent to the fact that, given arbitrary states $i, j \in V$, there exists a path from $i$ to $j$ respecting the edge orientation.

Denoting by $P_{i}(t)$ the probability that the Markov chain is at state $i$ at time $t$, the time evolution of $P_{i}(t)$ is given by the equation

$$
\frac{d}{d t} P_{i}(t)=\sum_{j: j \neq i}\left[P_{j}(t) w_{j i}(t)-P_{i}(t) w_{i j}(t)\right]
$$

The asymptotic properties related to this equation are as follows (cf. e.g. [5] for details). In the long time limit, $P_{i}(t)$ tends to an invariant time-periodic distribution $\pi_{i}(t)=\pi_{i}(t+\tau)$. The distribution $\pi(t)$ can be characterized as the unique invariant distribution of the discrete-time Markov chain $(X(t+n \tau))_{n>0}$. Other important quantities are the asymptotic elementary flow $\mathcal{Q}_{i j}(t)$ and current $\mathcal{J}_{i j}(t)$ along the edge $(i, j)$, which are given by

$$
\left\{\begin{array}{l}
\mathcal{Q}_{i j}(t):=\pi_{i}(t) w_{i j}(t), \\
\mathcal{J}_{i j}(t):=\pi_{i}(t) w_{i j}(t)-\pi_{j}(t) w_{j i}(t)=\mathcal{Q}_{i j}(t)-\mathcal{Q}_{j i}(t) .
\end{array}\right.
$$

Note that $\pi_{i}(t)>0$ for all $t>0$ and $i \in V$. Moreover $\mathcal{Q}_{i j}(t)>0$ for all $t>0$ if $(i, j) \in E$, while $\mathcal{Q}_{i j}(t)=0$ for all $t>0$ if $(i, j) \notin E$.

From equation (2.1) we get the continuity equation

$$
\partial_{t} \pi_{i}(t)+\sum_{j: j \neq i} \mathcal{Q}_{i j}(t)-\sum_{j: j \neq i} \mathcal{Q}_{j i}(t)=0 \quad \forall i \in V,
$$

which is equivalent to

$$
\partial_{t} \pi_{i}(t)+\sum_{j: j \neq i} \mathcal{J}_{i j}(t)=0 \quad \forall i \in V .
$$

The continuity equation (2.3) can be rewritten with a div operator in the form

$$
\partial_{t} \pi(t)+\operatorname{div} \mathcal{Q}(t)=0,
$$

where $\pi(t)$ and $\operatorname{div} \mathcal{Q}(t)$ are vectors with components $\pi_{i}(t)$ and $\operatorname{div}_{i} \mathcal{Q}(t):=\sum_{j} \mathcal{Q}_{i j}(t)-$ $\sum_{j} \mathcal{Q}_{j i}(t)$

Time independent transition rates $w_{i j}(t)=w_{i j}$ correspond to a particular case of our theory. In this case, we have a steady state characterized by the asymptotic distribution $\pi$, which fulfills the continuity equation

$$
\sum_{j: j \neq i} \mathcal{Q}_{i j}-\sum_{j: j \neq i} \mathcal{Q}_{j i}=0 \quad \forall i \in V
$$

where $\mathcal{Q}_{i j}=\pi_{i} w_{i j}$.

Finally, when the graph $(V, E)$ contains an edge $(i, j)$ if and only if it contains the edge $(j, i)$, we denote by $\sigma$ the average entropy production rate. In particular, we have

$$
\sigma=\frac{1}{2} \sum_{(i, j) \in E} \frac{1}{\tau} \int_{0}^{\tau} \mathcal{J}_{i j}(t) \ln \frac{\mathcal{Q}_{i j}(t)}{\mathcal{Q}_{j i}(t)} d t
$$


When the transition rates are time-independent, the above identity simply reads

$$
\sigma=\frac{1}{2} \sum_{(i, j) \in E} \mathcal{J}_{i j} \ln \frac{\mathcal{Q}_{i j}}{\mathcal{Q}_{j i}}
$$

Let us introduce the notations for time average and scalar products used in this paper. In what follows, when referring to a time-periodic function $f(t)$, we understand that its period equals $\tau$. Moreover, we denote by $\bar{f}$ the average of $f$ over a period, i.e.

$$
\bar{f}:=\frac{1}{\tau} \int_{0}^{\tau} f(t) d t
$$

The scalar product of two vectors $a(t)$ and $b(t)$ with entries parameterized by $i \in V$ is given by

$$
\langle a(t), b(t)\rangle:=\sum_{i \in V} a_{i}(t) b_{i}(t) ;
$$

while, if $a(t)$ and $b(t)$ are matrixes with entries parameterized by $(i, j) \in V \times V$, their scalar product is given by

$$
\langle a(t), b(t)\rangle:=\sum_{(i, j) \in V \times V} a_{i j}(t) b_{i j}(t) .
$$

Finally, in what follows Markov chains will always be considered as time-continuous (i.e. as Markov jump processes), also when not explicitly stated.

2.2. Empirical functionals. We describe now the class of empirical functionals on which we will focus and state the associated large deviation principle. Given a time-periodic matrix $\alpha(t)=\left(\alpha_{i j}(t):(i, j) \in V \times V\right)$ and a time-periodic vector $\gamma(t)=\left(\gamma_{i}(t): i \in V\right)$ we consider the empirical functional $Y_{\alpha, \gamma}^{(n)}$ defined as

$$
Y_{\alpha, \gamma}^{(n)}:=\frac{1}{n \tau} \sum_{\substack{t \in(0, n \tau]: \\ X(t-) \neq X(t+)}} \alpha_{X(t-), X(t+)}(t)+\frac{1}{n \tau} \int_{0}^{n \tau} \gamma_{X(t)}(t) d t .
$$

For example, if all components of $\gamma(t)$ are zero and the increments $\alpha_{i j}(t)$ are antisymmetric, i.e. $\alpha_{i j}(t)=-\alpha_{j i}(t)$, then $Y_{\alpha, \gamma}^{(n)}$ is a current, which is a key observable in stochastic thermodynamics. If the components of $\alpha(t)$ are zero, the component $\gamma_{i}(t)=1$ and the other components of $\gamma(t)$ are zero, then $Y_{\alpha, \gamma}^{(n)}$ is the fraction of time spent in state $i$.

Note that, as $n \rightarrow \infty, Y_{\alpha, \gamma}^{(n)}$ has the following asymptotics (cf. [5, Proposition 7.3]):

$$
Y_{\alpha, \gamma}^{(n)} \rightarrow y_{\alpha, \gamma}:=\overline{\langle\alpha, \mathcal{Q}\rangle}+\overline{\langle\gamma, \pi\rangle} .
$$

In particular, if $\alpha_{i j}=\ln \left(w_{i j} / w_{j i}\right)$ and $\gamma=0$, then $y_{\alpha, \gamma}$ equals the average entropy production rate $\sigma$ in (2.7).

As a byproduct of the large deviation (LD) principle given by [5, Theorem 2] and the contraction principle (cf. e.g. [14, 15, 25, 46]), $Y_{\alpha, \gamma}^{(n)}$ satisfies an LD principle as $n \rightarrow \infty$ with speed $n \tau$. Calling $I_{\alpha, \gamma}$ its rate functional, roughly it holds

$$
\mathbb{P}\left(Y_{\alpha, \gamma}^{(n)} \approx y\right) \asymp e^{-n \tau I_{\alpha, \gamma}(y)}, \quad y \in \mathbb{R}, n \gg 1 .
$$


We point out that $I_{\alpha, \gamma}(y) \geq 0$ and $I_{\alpha, \gamma}(y)=0$ if and only if $y=y_{\alpha, \gamma}$. This corresponds to the fact that $y_{\alpha, \gamma}$ is the typical value and different values of the functional are exponentially unlikely.

To describe the variational characterization of the LD rate functional $I_{\alpha, \gamma}$, we introduce the function $\Phi(q, p)$ defined for $q, p \geq 0$ as

$$
\Phi(q, p):=q \ln (q / p)-q+p,
$$

with the convention that $\Phi(0, p):=p$ and $\Phi(q, 0)=+\infty$ for $q>0$. Then, it holds

$$
I_{\alpha, \gamma}(y)=\inf \left\{I(Q, \rho):(Q, \rho) \in \mathcal{F}_{\alpha, \gamma, y}\right\},
$$

where

$$
I(Q, \rho):=\sum_{(i, j) \in E} \overline{\Phi\left(Q_{i j}(t), \rho_{i}(t) w_{i j}(t)\right)}
$$

and $\mathcal{F}_{\alpha, \gamma, y}$ denotes the family of pairs $(Q, \rho)=(Q(t), \rho(t))_{t \geq 0}$ such that

(i) $Q(t)$ is a time-periodic flow, i.e. $Q(t)=Q(t+\tau)$ and $Q(t)$ is a non-negative function on $V \times V$ which is zero outside $E$ for each time $t$;

(ii) $\rho(t)$ is a time-periodic probability measure on $V$;

(iii) the continuity equation $\partial_{t} \rho(t)+\operatorname{div} Q(t)=0$ is satisfied, where $\operatorname{div}_{i} Q(t):=$ $\sum_{j} Q_{i j}(t)-\sum_{j} Q_{j i}(t)$

(iv) $y=\overline{\langle\alpha, Q\rangle}+\overline{\langle\gamma, \rho\rangle}$.

We point out that one recovers from (2.13) that $I_{\alpha, \gamma}\left(y_{\alpha, \gamma}\right)=0$ since, denoting by $\mathcal{Q}=(\mathcal{Q}(t))_{t \geq 0}$ and $\pi=(\pi(t))_{t \geq 0}$ the asymptotic flow and density, respectively, it holds $I(\mathcal{Q}, \pi)=0$ in addition to (2.10).

Formula (2.14) corresponds to the joint LD rate functional of the empirical flow and measure. To recall their definition, given $t \geq 0$ we denote by $[t]$ the only number in $[0, \tau)$ such that $t-[t]$ is a multiple of $\tau$. Then the empirical flow $Q^{(n)}$ is defined as the measure on $E \times[0, \tau)$ given by

$$
Q^{(n)}(i, j, A):=\frac{1}{n} \sharp\{t \in(0, n \tau]: X(t-)=i, X(t+)=j,[t] \in A\},
$$

where $\sharp$ denotes the cardinality of the set. On the other hand, the empirical measure $\rho^{(n)}$ is defined as the measure on $V \times[0, \tau)$ such that

$$
\rho^{(n)}(i, A):=\frac{1}{n} \int_{0}^{n \tau} \mathbb{1}(X(t)=i,[t] \in A) d t,
$$

where $\mathbb{1}(\cdot)$ denotes the characteristic function (i.e. the function equals 1 if the event under consideration takes place, otherwise it equals zero). Note that, given a time-periodic flow $Q=(Q(t))_{t \geq 0}$, we can think of $Q$ as the measure on $E \times$ $[0, \tau)$ with weights $(i, j, d t) \mapsto Q_{i j}(t) d t$. Given a time-periodic probability measure $\rho=(\rho(t))_{t \geq 0}$ on $V$ we can think of $\rho$ as the measure on $V \times[0, \tau)$ with weights $(i, d t) \mapsto \rho_{i}(t) d t$. In [5, Theorem 2] it is proved that the pair $\left(Q^{(n)}, \pi^{(n)}\right)$ satisfies a LD principle with speed $n \tau$ and rate functional $I(Q, \rho)$ given by (2.14) if $(Q, \rho)=$ $(Q(t), \rho(t))_{t \geq 0}$ satisfies the above conditions (i), (ii), (iii). If these conditions are not fulfilled, then $I(Q, \rho)$ equals infinity. Since

$$
Y_{\alpha, \gamma}^{(n)}=\frac{1}{\tau} \sum_{i, j} \int_{[0, \tau)} \alpha_{i j}(t) Q^{(n)}(i, j, d t)+\frac{1}{\tau} \sum_{i} \int_{[0, \tau)} \gamma_{i}(t) \rho^{(n)}(i, d t),
$$

(2.13) follows from the contraction principle and the above LD principle for $\left(Q^{(n)}, \pi^{(n)}\right)$. 
The asymptotic diffusion coefficient $D_{\alpha, \gamma}$ associated with $Y_{\alpha, \gamma}^{(n)}$ is defined as

$$
2 D_{\alpha, \gamma}:=\lim _{n \rightarrow \infty} n \tau \operatorname{Var}\left(Y_{\alpha, \gamma}^{(n)}\right) .
$$

This quantity can be obtained from the rate functional $I_{\alpha, \gamma}$ by the identity

$$
2 D_{\alpha, \gamma}=\frac{1}{I_{\alpha, \gamma}^{\prime \prime}\left(y_{\alpha, \gamma}\right)}
$$

where $I_{\alpha, \gamma}^{\prime \prime}$ denotes the second derivative of $I_{\alpha, \gamma}$. We point out that in the mathematical literature the asymptotic diffusion coefficient is defined without the factor 2 in the l.h.s. of (2.16).

Formula (2.17) can be applied when the rate function $I_{\alpha, \gamma}$ is twice differentiable around its minimum point $y_{\alpha, \gamma}$. If the set $\mathcal{F}_{\alpha, \gamma, y}$ defined after (2.14) is non-empty for any real value $y$, then the differentiability could be proved using the smoothness of the function (2.12) on points with strictly positive coordinates and the linearity of the constraint $(i v)$ in the definition of $\mathcal{F}_{\alpha, \gamma, y}$. There are however exceptional cases when this does not happen. As an example consider the case when $\gamma \equiv 0$ and $\alpha_{i j}=f_{j}-f_{i}$ for fixed time-independent constants $\left(f_{i}\right)_{i \in V}$. Given $(Q, \rho) \in \mathcal{F}_{\alpha, \gamma, y}$ we deduce that $\operatorname{div} \bar{Q}=0$ by integrating the continuity equation $\partial_{t} \rho(t)+\operatorname{div} Q(t)=0$ on a period and using that $\rho(t)$ is periodic. Due to the gradient representation $\alpha_{i j}=f_{i}-f_{j}$ and since $\operatorname{div} \bar{Q}=0$, by a discrete integration by parts we obtain that $\langle\alpha, \bar{Q}\rangle=0$. We get therefore that $\mathcal{F}_{\alpha, \gamma, y}=\emptyset$ for any $y \neq 0$ (indeed, property (iv) in the definition of $\mathcal{F}_{\alpha, \gamma, y}$ cannot be fulfilled for $y \neq 0$ ). As a consequence $I_{\alpha, \gamma}(y)=+\infty$ for $y \neq 0$ and $I_{\alpha, \gamma}(0)=0$, hence $I_{\alpha, \gamma}$ is not differentiable. In this case formula (2.17) cannot be applied. See also Remark 6.1 for another exceptional class.

\section{MAIN RESUlts}

In this section we present our main GTURs, given by inequalities (GTUR 1), (GTUR 2),..., GTUR 7) below. Apart from (GTUR 7), which is a generalization of the result derived in [42], their derivation is obtained by extending the methods and ideas from [1. In particular, in Sections 5 and 6 we provide general methods to get GTUR's for $\alpha$ generic and $\alpha$ antisymmetric, respectively (cf. Theorem 1, 2 and 3). The results (GTUR 1), (GTUR 3) and (GTUR 5) presented below are a special case of a class of GTUR's obtained in Corollaries 5.2, 6.2 and 6.6, respectively. Therefore, we refer to Sections 5 and 6 for more results and proofs. The extension of the GTUR from 42 to asymmetric protocols is provided in Section 7 (cf. Theorem 4).

From now on, without further mention, we restrict to the case that the asymptotic value $y_{\alpha, \gamma}$ of the empirical functional $Y_{\alpha, \gamma}^{(n)}$ is non zero (see Remark 5.1 for the case $\left.y_{\alpha, \gamma}=0\right)$.

3.1. GTUR with generic increments. From Corollary [5.2, which contains a more general result, we obtain:

GTUR 1. If the increments $\alpha$ are time-independent (i.e. $\alpha_{i, j}(t) \equiv \alpha_{i, j}$ ) and $\gamma \equiv 0$, then

$$
\frac{D_{\alpha, 0}}{y_{\alpha, 0}^{2}} \geq \frac{1}{\widehat{\sigma}},
$$


where

$$
\widehat{\sigma}:=2 \sum_{(i, j) \in E}\left(\overline{\mathcal{Q}}_{i j}\right)^{2} \overline{\frac{1}{\mathcal{Q}_{i j}}} .
$$

For the case of time-homogeneous Markov chains, $\widehat{\sigma}=2 \sum_{(i, j) \in E} \mathcal{Q}_{i j}$, and this GTUR becomes [19, Eq. (19)]. Hence, (GTUR 1) is a generalization of this inequality to time-periodic Markov chains. The quantity $\sum_{(i, j) \in E} \mathcal{Q}_{i j}$, which is the rate of average number of transitions, is known as dynamical activity. For time-periodic Markov chains, due to Jensen's inequality, we have the bound $\widehat{\sigma} \geq 2 \sum_{(i, j) \in E} \overline{\mathcal{Q}}_{i j}$, i.e. $\widehat{\sigma} / 2$ is larger than the dynamical activity.

From Corollary 5.3 we obtain:

GTUR 2. For generic increments $\alpha$, it holds

$$
\frac{D_{\alpha, \gamma}}{y_{\alpha, \gamma}^{2}} \geq \frac{1}{C(p)}
$$

where $p=\left(p_{i}\right)_{i \in V}$ is any probability on $V$ with $\langle\bar{\gamma}, p\rangle=0$ and

$$
C(p):=2 \sum_{(i, j) \in E} p_{i}^{2} \overline{\left(\frac{w_{i j}^{2}}{\mathcal{Q}_{i j}}\right)}=2 \sum_{(i, j) \in E} p_{i}^{2} \overline{\left(\frac{w_{i j}}{\pi_{i}}\right)} .
$$

Note that the above probability $p$ is time-independent. This novel GTUR is valid for generic linear functionals of the form (2.9), including the case $\alpha=0$, which corresponds to functionals that depend only on the empirical density.

3.2. GTUR with antisymmetric increments. In this subsection we assume, without further mention, that

$$
(y, z) \in E \Leftrightarrow(z, y) \in E .
$$

For the particular case of antisymmetric increments $\alpha_{i, j}(t)=-\alpha_{j, i}(t)$, we have the following GTUR's.

First, from Corollary 6.2, which contains a more general result, we obtain:

GTUR 3. If $\alpha$ is time-independent and antisymmetric and $\gamma \equiv 0$, then

$$
\frac{D_{\alpha, 0}}{y_{\alpha, 0}^{2}} \geq \frac{1}{\widetilde{\sigma}},
$$

where

$$
\widetilde{\sigma}:=\sum_{(i, j) \in E}\left(\overline{\mathcal{J}}_{i j}\right)^{2} \overline{\frac{1}{\mathcal{Q}_{i j}+\mathcal{Q}_{j i}}} .
$$

This GTUR corresponds to the first bound in [1, Eq. (27)].

Second, from Corollary 6.3, we obtain:

GTUR 4. For generic antisymmetric increments $\alpha$, it holds

$$
\frac{D_{\alpha, \gamma}}{y_{\alpha, \gamma}^{2}} \geq \frac{1}{C_{\mathrm{a}}(p)},
$$

where $p=\left(p_{i}\right)_{i \in V}$ is any probability on $V$ with $\langle\bar{\gamma}, p\rangle=0$ and

$$
C_{\mathrm{a}}(p):=\sum_{(i, j) \in E} \overline{\left(\frac{\left(p_{i} w_{i j}-p_{j} w_{j i}\right)^{2}}{\mathcal{Q}_{i j}+\mathcal{Q}_{j i}}\right)} .
$$


Third, from Corollary 6.6, which contains a more general result, we obtain:

GTUR 5. If $\alpha$ is time-independent and antisymmetric and $\gamma \equiv 0$, then

$$
\frac{D_{\alpha, 0}}{y_{\alpha, 0}^{2}} \geq \frac{1}{\sigma^{*}}
$$

where

$$
\sigma^{*}:=\frac{1}{2} \sum_{(i, j) \in E}\left(\overline{\mathcal{J}}_{i j}\right)^{2} \overline{\left(\frac{1}{\mathcal{J}_{i j}} \ln \frac{\mathcal{Q}_{i j}}{\mathcal{Q}_{j i}}\right)} .
$$

This GTUR corresponds to the second bound in [1, Eq. (27)]. Furthermore, due to the inequality $\sigma^{*} \geq \widetilde{\sigma}$, which has been proved in [1, GTUR 5) can be also derived directly from (GTUR 3). The original TUR for time-homogeneous Markov chains is a particular case of (GTUR 5). For a time-homogeneous Markov chain $\sigma^{*}$ becomes the average entropy production rate $\sigma$ in (2.8).

Fourth, from Corollary 6.7, we obtain:

GTUR 6. For generic antisymmetric increments $\alpha$, it holds

$$
\frac{D_{\alpha, \gamma}}{y_{\alpha, \gamma}^{2}} \geq \frac{1}{C_{\mathrm{a}}^{*}(p)},
$$

where $p=\left(p_{i}\right)_{i \in V}$ is any probability on $V$ with $\langle\bar{\gamma}, p\rangle=0$ and

$$
C_{\mathrm{a}}^{*}(p):=\frac{1}{2} \sum_{(i, j) \in E} \overline{\left(\frac{\left(p_{i} w_{i j}-p_{j} w_{j i}\right)^{2}}{\mathcal{J}_{i j}}\right) \ln \frac{\mathcal{Q}_{i j}}{\mathcal{Q}_{j i}}} .
$$

This GTUR, for the particular case $\bar{\gamma}=0$ (which is equivalent to the fact that $\gamma_{i}(t)=\frac{d}{d t} g_{i}(t)$ for periodic functions $\left.g_{i}\right)$, has been obtained in [27] with a different derivation (cf. [27, Eq. (14),(15),(16)]).

The inequality (GTUR 6) can be derived by the general method presented in Theorem 3 as well as directly from (GTUR 4) by the bound (6.30) presented in Section 6.5.

3.3. GTUR with naive entropy production. Our last GTUR follows from Theorem 4, which contains more general results:

GTUR 7. If $\alpha_{i, j}(t)=-\alpha_{j, i}(\tau-t)$ and $\gamma_{i}(t)=-\gamma_{i}(\tau-t)$ for any $i, j$ and $t \in[0, \tau]$, then

$$
\frac{D_{\alpha, \gamma}}{y_{\alpha, \gamma}^{2}} \geq \frac{\tau}{e^{\tau \sigma_{\text {naive }}-1}}
$$

where

$$
\begin{aligned}
\sigma_{\text {naive }} & :=\frac{1}{\tau} \sum_{(i, j) \in E} \int_{0}^{\tau} \pi_{i}(s)\left[w_{i j}(\tau-s)-w_{i j}(s)\right] d s \\
& +\frac{1}{\tau} \sum_{(i, j) \in E} \int_{0}^{\tau} \pi_{i}(s) w_{i j}(s) \ln \frac{w_{i j}(s)}{w_{j i}(\tau-s)} d s .
\end{aligned}
$$

The above result is a generalisation to arbitrary protocols of [42, Eq. (2)] for the empirical functionals $Y_{\alpha, \gamma}^{(n)}$. When the period $\tau$ is small, the inverse rate given by the r.h.s. of (GTUR 7) is well approximated by $1 / \sigma_{\text {naive }}$. Note that for symmetric protocols $\sigma_{\text {naive }}=\sigma$. In the limit $\tau \rightarrow 0$ (GTUR 7) can be applied only to currents 


\begin{tabular}{|c|c|c|}
\hline GTUR & Lower bound & Restrictions on $Y_{\alpha, \gamma}^{(n)}$ \\
\hline GTUR 1 & $1 / \widehat{\sigma}$ & $\alpha_{i j}(t)=\alpha_{i j}$ and $\gamma_{i}(t)=0$ \\
\hline GTUR 2 & $1 / C(p)$ & $\langle\bar{\gamma}, p\rangle=0$ \\
\hline GTUR 3 & $1 / \widetilde{\sigma}$ & $\alpha_{i j}(t)=\alpha_{i j}, \alpha_{i j}=-\alpha_{j i}$ and $\gamma_{i}(t)=0$ \\
\hline GTUR 4 & $1 / C_{a}(p)$ & $\alpha_{i j}(t)=-\alpha_{j i}(t)$ and $\langle\bar{\gamma}, p\rangle=0$ \\
\hline GTUR 5 & $1 / \sigma^{*}$ & $\alpha_{i j}(t)=\alpha_{i j}, \alpha_{i j}=-\alpha_{j i}$ and $\gamma_{i}(t)=0$ \\
\hline GTUR 6 & $1 / C_{a}^{*}(p)$ & $\alpha_{i j}(t)=-\alpha_{j i}(t)$ and $\langle\bar{\gamma}, p\rangle=0$ \\
\hline GTUR 7 & $\tau /\left(e^{\tau \sigma_{\text {naive }}}-1\right)$ & $\alpha_{i j}(t)=-\alpha_{j i}(\tau-t)$ and $\gamma_{i}(t)=-\gamma_{i}(\tau-t)$ \\
\hline
\end{tabular}

TABLE 1. Summary of GTUR's written as $D_{\alpha, \gamma} / y_{\alpha, \gamma}^{2} \geq$ lower bound. The GTUR's are valid for the linear functionals $Y_{\alpha, \gamma}^{(n)}$ that fulfill the conditions on the third column.

with time-independent increments (due to the constraints $\alpha_{i, j}(t)=-\alpha_{j, i}(\tau-t$ ) and $\left.\gamma_{i}(t)=-\gamma_{i}(\tau-t)\right)$ and it reduces to the classical thermodynamic uncertainty relation $D_{\alpha, 0} / y_{\alpha, 0}^{2} \geq 1 / \sigma$.

3.4. Optimization and comparisons. In this subsection we show three propositions. The first is concerned with the optimal $p$ in the universal rate $C(p)$ in (GTUR 2). The second is concerned with the relation between (GTUR 2), (GTUR 4) and (GTUR 6). The third is concerned with the relation between (GTUR 1), (GTUR 3) and (GTUR 5).

We recall that (GTUR 2) holds for any choice of the increments $\alpha$, antisymmetric or not. The following result shows the optimal bound that can be obtained in (GTUR 2) by taking the minimum among $p=\left(p_{i}\right)_{i \in V}$ of $C(p)$ :

Proposition 3.1. Setting

$$
A_{i}:=\left[2 \sum_{j:(i, j) \in E} \overline{\left(\frac{w_{i j}}{\pi_{i}}\right)}\right]^{-1},
$$

the optimal bound in (GTUR 2) is the following:

(i) if $\bar{\gamma}=0$, then

$$
\frac{D_{\alpha, \gamma}}{y_{\alpha, \gamma}^{2}} \geq \sum_{i} A_{i}
$$

(ii) if $\bar{\gamma} \neq 0$ and $\bar{\gamma}$ has neither all entries positive nor all entries negative, then

$$
\frac{D_{\alpha, \gamma}}{y_{\alpha, \gamma}^{2}} \geq \frac{\left(\sum_{i} A_{i}\right)\left(\sum_{i} A_{i} \bar{\gamma}_{i}^{2}\right)-\left(\sum_{i} A_{i} \bar{\gamma}_{i}\right)^{2}}{\sum_{i} A_{i} \bar{\gamma}_{i}^{2}},
$$

and the r.h.s. of (3.10) is a positive number.

For the proof of the above proposition see Appendix $\mathrm{A}$. We point out that the optimization among $p=\left(p_{i}\right)_{i \in V}$ for the other constants $C_{\mathrm{a}}(p)$ and $C_{\mathrm{a}}^{*}(p)$ appearing in (GTUR 4) and (GTUR 6), respectively, cannot be solved explicitly in the general case.

Remark 3.2. When the increments $\alpha$ are time-independent and $\gamma \equiv 0$, one can apply both (GTUR 1) and the optimal (GTUR 2) given by (3.9). If the asymptotic density $\pi(t)$ is time-independent as in the time-homogeneous case, or as in the 


\begin{tabular}{|c|c|}
\hline SET-UP & HIERARCHY OF GTUR's \\
\hline \hline $\begin{array}{l}\pi_{i}(t)=\pi_{i} \\
\alpha_{i j}(t)=\alpha_{i j} \\
\gamma_{i}(t)=0\end{array}$ & Optimal GTUR2 (3.9) $\Rightarrow$ (GTUR 1) \\
\hline$\alpha_{i j}(t)=-\alpha_{j i}(t)$ & $\left\{\begin{array}{l}\text { (GTUR 4 } \\
\text { (GTUR 4 }\end{array} \Rightarrow\right.$ (GTUR 2
\end{tabular}

TABLE 2. Implications between GTUR's

time-periodic random walk on the ring considered in Section 4.2, we can prove that (3.9) is stronger than (GTUR 1). We refer to Appendix A for the derivation.

When $\alpha$ is antisymmetric, we can apply three $p$-dependent GTUR's, i.e. (GTUR 2), GTUR 4 and GTUR 6). Indeed, GTUR 4) is the optimal one as follows from the next result:

Proposition 3.3. Assume that $(i, j) \in E$ if and only if $(j, i) \in E$. Then for each probability measure $p=\left(p_{i}\right)_{i \in V}$ it holds $C_{a}^{*}(p) \geq C_{a}(p)$ and $C(p) \geq C_{a}(p)$. In particular, when $\alpha$ is antisymmetric, GTUR 4 provides the optimal lower bound of $D_{\alpha, \gamma} / y_{\alpha, \gamma}^{2}$ between (GTUR 2), (GTUR 4) and (GTUR 6).

For the proof of the above proposition see Appendix A. The optimality of (GTUR 4) stated in Proposition 3.3 is also a consequence of a special alternative derivation of this bound by an optimization procedure (cf. Remark 6.4).

Similarly to Proposition 3.3 we have the following result for the universal constants in (GTUR 1), (GTUR 3) and (GTUR 5):

Proposition 3.4. It holds $\sigma^{*} \geq \widetilde{\sigma}$ and $\widehat{\sigma} \geq \widetilde{\sigma}$. In particular, when $\alpha$ is antisymmetric and time-independent and $\gamma \equiv 0$, (GTUR 3) provides the optimal lower bound between (GTUR 1), (GTUR 3) and (GTUR 5).

For the proof of the above proposition see Appendix $\mathrm{A}$ (we recall that the bound $\sigma^{*} \geq \widetilde{\sigma}$ has been derived in [1]).

In Section 4.2, considering the case of a random walk on the discrete ring, we show that the optimal bound (GTUR 4) of Proposition 3.3 and the optimal bound (GTUR 3 of Proposition 3.4 are non-comparable bounds. Similarly the bounds (GTUR 4) and (GTUR 7) are non-comparable, as well as the bounds (GTUR 3) and (GTUR 7). This is illustrated in Figure1 in Section 4.2 and corresponds to the crossings of the plotted curves.

We collect some of the above comparative results in Table 2 ,

3.5. Further comments on (GTUR 7). The rate $\sigma_{\text {naive }} \geq 0$ is the asymptotic average value per unit time of the functional of the trajectories introduced in 5, Section 4] and described as follows: the functional equals the logarithm of the ratio of the weight of the forward trajectory to the weight of the backward trajectory, 
without reversal of the protocol. This situation is different from the average entropy production rate $\sigma \geq 0$, for which the reversed trajectory with reversed protocol is considered. Furthermore, the quantity $\sigma_{\text {naive }}$ can be written as $\sigma_{\text {naive }}=\sigma+\sigma_{\text {asy }}$, where

$$
\sigma_{\text {asy }}:=\sum_{(i, j) \in E} \overline{\mathcal{Q}_{i j}\left(A_{i j}-1-\ln A_{i j}\right)},
$$

and $A_{i j}(t):=w_{i j}(\tau-t) / w_{i j}(t)$. This decomposition has a nice physical interpretation, the average entropy production rate $\sigma$ quantifies energy dissipation and $\sigma_{\text {asy }}$ is zero if the protocol is symmetric. We point out that $-\sigma \leq \sigma_{\text {asy }} \leq \sigma_{\text {naive }}$ and that $\sigma_{\text {asy }}$ can have arbitrary sign, as demonstrated with an explicit calculation in Section 4.2 .

If, in addition to (GTUR 7), it is possible to apply (GTUR 3) or (GTUR 4) (for example for currents with time-independent increments), then there is a priori no fixed order between the corresponding rates. This fact is demonstrated by an example in Section 4.2.

Finally, GTUR 7) does not work well when the periodically driven Markov chain is obtained by a weak perturbation of a time-homogeneous Markov chain with continuous time. Indeed, suppose that the transition rates are given by $w_{i j}(t)=$ $c_{i j}+\varepsilon d_{i j}(t)$, where $c_{i j}$ are the transition rates of an irreducible time-homogeneous Markov chain and $d_{i j}(t)$ are genuinely time periodic, with period $\tau$. Then, when $\varepsilon \rightarrow 0$, the value in the r.h.s. of (GTUR 7) converges to $\tau\left(e^{\tau \sigma}-1\right)^{-1}$, which is smaller (and even much smaller for $\tau$ large) than $1 / \sigma$ entering in the standard thermodynamic uncertainty relation. On the other hand, the rates $C(p), C_{\mathrm{a}}(p), C_{\mathrm{a}}\left(p^{*}\right)$, $\widehat{\sigma}, \widetilde{\sigma}, \sigma^{*}$ behave well under perturbations.

3.6. Comments on the weights $\gamma$. We observe that (GTUR 2), GTUR 4) and GTUR 6) are uniform among the weights $\gamma$ such that $\langle\gamma, p\rangle=0$ for some probability measure $p$ on $V$. We remark that one cannot find a GTUR uniform among all $\gamma$ 's. Indeed, if we consider new weights $\gamma^{\prime}$ defined as $\gamma_{i}^{\prime}=\gamma_{i}+c$ for some fixed constant $c$, we get $y_{\alpha, \gamma^{\prime}}=y_{\alpha, \gamma}+c$, while $D_{\alpha, \gamma^{\prime}}=D_{\alpha, \gamma}$. In particular the ratio of the asymptotic diffusion coefficient to the squared asymptotic value can be made arbitrarily small by playing with $c$. On the other hand, if we take $\gamma^{\prime}=c \gamma$ for some $c \neq 0$, we have $y_{\alpha, \gamma^{\prime}}=c y_{\alpha, \gamma}$, while $D_{\alpha, \gamma}=c^{2} D_{\alpha, \gamma^{\prime}}$, thus implying that $D_{\alpha, \gamma} / y_{\alpha, \gamma}^{2}=D_{\alpha, \gamma^{\prime}} / y_{\alpha, \gamma^{\prime}}^{2}$. As a consequence GTUR's are automatically uniform among proportional $\gamma$ 's. In (GTUR 2), (GTUR 4) and (GTUR 6) one goes further replacing proportionality by the weaker condition $\langle\bar{\gamma}, p\rangle=0$.

We also observe that, given $\gamma$, the existence of a probability measure $p$ such that $\langle\bar{\gamma}, p\rangle=0$ is equivalent to the fact that the entries of $\bar{\gamma}$ are not all positive and not all negative. If for example the entries of $\gamma$ are all positive, by taking a suitable constant $c$ one can apply (GTUR 2), (GTUR 4) and (GTUR 6) to the weights $\alpha, \gamma^{\prime}$ where $\gamma_{i}^{\prime}=\gamma_{i}+c$, and then recover information on $Y_{\alpha, \gamma}^{(n)}$ by using that $Y_{\alpha, \gamma}^{(n)}=Y_{\alpha, \gamma^{\prime}}^{(n)}-c$, $y_{\alpha, \gamma}=y_{\alpha, \gamma^{\prime}}-c, D_{\alpha, \gamma}=D_{\alpha, \gamma^{\prime}}$.

3.7. Comments on the derivation of the GTUR's. We comment the methods used to derive the above GTUR's, which are summarized in Table 1;

(1) Due to (2.13) we have the upper bound

$$
I_{\alpha, \gamma}(y) \leq I(Q, \rho) \text { for any }(Q, \rho) \in \mathcal{F}_{\alpha, \gamma, y},
$$


where $I(Q, \rho)$ is the explicit function given in (2.14) and the set $\mathcal{F}_{\alpha, \gamma, y}$ is defined after (2.14).

(2) We assume to have an $y$-parameterized pair $\left(Q_{y}, \rho_{y}\right) \in \mathcal{F}_{\alpha, \gamma, y}$ such that $\left(Q_{y}, \rho_{y}\right)$ differs from $(\mathcal{Q}, \pi)$ by a term proportional to $y-y_{\alpha, \gamma}$.

(3) Plugging the above $y$-parameterized pair $\left(Q_{y}, \rho_{y}\right)$ in the inequality (3.12) and taking a 2nd order Taylor expansion of the explicit function $y \mapsto I\left(Q_{y}, \rho_{y}\right)$ around $y_{\alpha, \gamma}$, one gets a quadratic local bound of $I_{\alpha, \gamma}$ at $y_{\alpha, \gamma}$ (cf. Theorem 1 in Section 5). A lower bound for the ratio $D_{\alpha, \gamma} / y_{\alpha, \gamma}^{2}$ can then be obtained by (2.17).

(4) By exhibiting different choices of $\left(Q_{y}, \rho_{y}\right)$ satisfying the above general conditions, we obtain (GTUR 1) and (GTUR 2).

(5) When $\alpha$ is antisymmetric $Y_{\alpha, \gamma}^{(n)}$ can be expressed as a linear function of the empirical density and current, whose LD principle has been derived in [5] with an explicit LD rate functional $I_{*}$. The above strategy can be implemented working with currents $(J)$ instead of flows $(Q)$. Hence, we get a general result given by Theorem 2, which is the analogous of Theorem 1. Indeed, by a different approach, we show that Theorem 2 can be even derived from Theorem 1. By exhibiting different choices of $\left(J_{y}, \rho_{y}\right)$ satisfying our general conditions, we get (GTUR 3) and (GTUR 4).

(6) GTUR 6) is a consequence of a general result detailed in Theorem 3 . This theorem can be obtained along the above scheme, with the exception that one uses an upper bound of the LD rate function $I_{*}$ by a suitable function proposed by [21] and afterwards applies a 2nd order Taylor expansion to this function. We also show that indeed Theorem 3 can also be obtained as corollary of Theorem 2 ,

(7) (GTUR 7) is a special case of a more general result given in Theorem 4 in Section 7 and its derivation follows very closely the one in [42]. The trajectory of the Markov chain on the time interval $[0, n \tau]$ can be thought of as a concatenation of paths on the fundamental periods $[0, \tau],[\tau, 2 \tau], .$. , $[(n-1) \tau, n \tau]$. One obtains a LD principle for the frequencies of these paths. On the other hand, the empirical functional $Y_{\alpha, \gamma}^{(n)}$ can be expressed as a linear functional of the above frequencies and by contraction one gets a new variational characterization for $I_{\alpha, \gamma}$. By playing with suitable inputs in the variational characterization, one finally gets the resulting quadratic local upper bounds on $Y_{\alpha, \gamma}^{(n)}$ and, as a byproduct with (2.17), (GTUR 7).

\section{EXAMPLES}

We study here two specific examples, given by a periodically driven 2-state Markov chain and a periodically driven random walk on a ring. The latter is particularly relevant for the comparison of GTUR's.

4.1. 2-state model. We consider a periodically driven 2-state Markov chain, which can be used e.g. to study a quantum dot. We take $V=\{0,1\}$. Then the periodic stationary distribution $\pi_{i}(t)$ has the following form (cf. [5, Sec. 6], [18, Prop. 3.13]): 


$$
\begin{aligned}
& \pi_{0}(t)=\frac{e^{-C(t)}}{1-e^{-C(\tau)}}\left[\int_{0}^{t} w_{10}(s) e^{C(s)} d s+e^{-C(\tau)} \int_{t}^{\tau} w_{10}(s) e^{C(s)} d s\right], \\
& \pi_{1}(t)=\frac{e^{-C(t)}}{1-e^{-C(\tau)}}\left[\int_{0}^{t} w_{01}(s) e^{C(s)} d s+e^{-C(\tau)} \int_{t}^{\tau} w_{01}(s) e^{C(s)} d s\right],
\end{aligned}
$$

where

$$
C(t):=\int_{0}^{t}\left[w_{01}(s)+w_{10}(s)\right] d s .
$$

For simplicity we restrict below to $\bar{\gamma}=0$.

When $\alpha$ is arbitrary, by Proposition 3.1 we get the optimal (among $p=\left(p_{0}, p_{1}\right)$ ) (GTUR 2)

$$
\frac{D_{\alpha, \gamma}}{y_{\alpha, \gamma}^{2}} \geq \frac{1}{2}\left[\left(\overline{w_{01} / \pi_{0}}\right)^{-1}+\left(\overline{w_{10} / \pi_{1}}\right)^{-1}\right]
$$

When $\alpha$ is antisymmetric, by Proposition 3.3 we know that (GTUR 4) is the optimal one between (GTUR 2), (GTUR 4) and (GTUR 6). One can optimize $C_{a}(p)$ among the probabilities $p=\left(p_{0}, p_{1}\right)$ as follows. Defining $T(t)$ as

$$
T(t):=\mathcal{Q}_{01}(t)+\mathcal{Q}_{10}(t)=\pi_{0}(t) w_{01}(t)+\pi_{1}(t) w_{10}(t),
$$

by straightforward computations we get

$$
\min \left\{C_{a}(p): p=\left(p_{0}, p_{1}\right)\right\}=2 \frac{\overline{w_{01}^{2} / T} \cdot \overline{w_{10}^{2} / T}-\left(\overline{w_{01} w_{10} / T}\right)^{2}}{\overline{\left(w_{01}+w_{10}\right)^{2} / T}} .
$$

Note that, if one introduces on the fundamental period $[0, \tau]$ the probability measure

$$
\nu(d t):=\left[\int_{0}^{\tau} \frac{1}{T(s)} d s\right]^{-1} \frac{1}{T(t)} d t,
$$

then we can think of $w_{01}(t)$ and $w_{10}(t)$ as random variables on the probability space $([0, \tau], \nu)$ and the optimal constant given by the r.h.s. of (4.2) equals

$$
2 \frac{\operatorname{Cov}_{\nu}\left(w_{01} ; w_{10}\right)}{\nu\left(\left(w_{01}+w_{10}\right)^{2}\right)}
$$

By (GTUR 4) we have, for $\alpha$ antisymmetric,

$$
\frac{D_{\alpha, \gamma}}{y_{\alpha, \gamma}^{2}} \geq \frac{1}{2} \frac{\overline{\left(w_{01}+w_{10}\right)^{2} / T}}{\overline{w_{01}^{2} / T} \cdot \overline{w_{10}^{2} / T}-\left(\overline{w_{01} w_{10} / T}\right)^{2}} .
$$

We recall that the GTUR's presented in Section 3 are meaningful under the condition that $y_{\alpha, \gamma} \neq 0$. If one restricts to time-independent currents (i.e. $\alpha_{i j}(t)=$ $\alpha_{i j}, \alpha_{i j}=-\alpha_{j i}$, and $\gamma \equiv 0$ ), then this condition fails, since $\overline{\mathcal{J}}_{01}=0$ for all $t \geq 0$ (see Remark 6.1 for a generalization). 
4.2. Random walk on the ring. We consider a random walk on a ring with $N$ sites, where $k_{+}(t)$ and $k_{-}(t)$ are the periodic probability rates to make a unitary jump clockwise and anticlockwise, respectively. In this case $\pi_{i}(t)=1 / N$ by symmetry.

Due to Proposition 3.1, when $\alpha$ is arbitrary and $\bar{\gamma}=0$, we have the optimal (GTUR 2)

$$
\frac{D_{\alpha, \gamma}}{y_{\alpha, \gamma}^{2}} \geq \frac{1}{2} \frac{1}{\bar{k}_{+}+\bar{k}_{-}}=: \frac{1}{r} .
$$

By Proposition 3.3, when $\alpha$ is antisymmetric and $\bar{\gamma}=0$, GTUR 4 is optimal among (GTUR 2), (GTUR 4) and (GTUR 6). By optimizing (GTUR 4) among the probability measures $p=\left(p_{i}\right)$, we get that the minimum is attained at the uniform probability and therefore we get the optimal (GTUR 4)

$$
\frac{D_{\alpha, \gamma}}{y_{\alpha, \gamma}^{2}} \geq \frac{1}{2}\left[\overline{\left(\frac{\left(k_{-}-k_{+}\right)^{2}}{k_{-}+k_{+}}\right)}\right]^{-1}=: \frac{1}{r_{\mathrm{a}}} .
$$

For this model we have

$$
\widetilde{\sigma}=2\left(\bar{k}_{-}-\bar{k}_{+}\right)^{2} \overline{\frac{1}{k_{-}+k_{+}}} .
$$

and

$$
\begin{aligned}
\sigma_{\text {naive }} & =\overline{k_{+} \ln \frac{k_{+}}{k_{-}(\tau-\cdot)}+k_{-} \ln \frac{k_{-}}{k_{+}(\tau-\cdot)}} \\
& =\sigma+\frac{k_{+} \ln \frac{k_{-}}{k_{-}(\tau-\cdot)}+k_{-} \ln \frac{k_{+}}{k_{+}(\tau-\cdot)}}{}
\end{aligned}
$$

Above the function $k_{ \pm}(\tau-\cdot)$ is defined as $t \mapsto k_{ \pm}(\tau-t)$. If we take for example $k_{+} \equiv 1$ we get $\sigma_{\text {naive }}=\sigma+\overline{\ln \frac{k_{-}}{k_{-}(\tau-\cdot)}}$. This shows that there is not a fixed order between $\sigma_{\text {naive }}$ and $\sigma$. Indeed, given a positive periodic function $f$, the random walk with rates $k_{+} \equiv 1$ and $k_{-}=f$ and the random walk with rates $k_{+} \equiv 1$ and $k_{-}=f(\tau-\cdot)$ have inverted ordering for $\sigma$ and $\sigma_{\text {naive. }}$. In particular, $\sigma_{\text {asy }}=\sigma_{\text {naive }}-\sigma$ can be positive and negative as well.

Let us now take the following time-symmetric protocol, where $a, b, c, d$ are positive numbers:

$$
k_{+}(t)=\left\{\begin{array}{ll}
a & \text { if } t \in[0, \tau / 4) \\
b & \text { if } t \in[\tau / 4,3 \tau / 4) \\
a & \text { if } t \in[3 \tau / 4, \tau)
\end{array} \quad \text { and } \quad k_{-}(t)= \begin{cases}c & \text { if } t \in[0, \tau / 4) \\
d & \text { if } t \in[\tau / 4,3 \tau / 4) \\
c & \text { if } t \in[3 \tau / 4, \tau)\end{cases}\right.
$$

Then we have

$$
\begin{aligned}
r & =a+b+c+d, \\
r_{\mathrm{a}} & =\frac{(a-c)^{2}}{a+c}+\frac{(b-d)^{2}}{b+d}, \\
\widetilde{\sigma} & =\frac{1}{4}[(a+b)-(c+d)]^{2}\left(\frac{1}{a+c}+\frac{1}{b+d}\right), \\
\sigma_{\text {naive }} & =\sigma=\frac{a-d}{2} \ln \frac{a}{d}+\frac{b-c}{2} \ln \frac{b}{c} .
\end{aligned}
$$

Note that all the above quantities do not depend on the period $\tau$. 


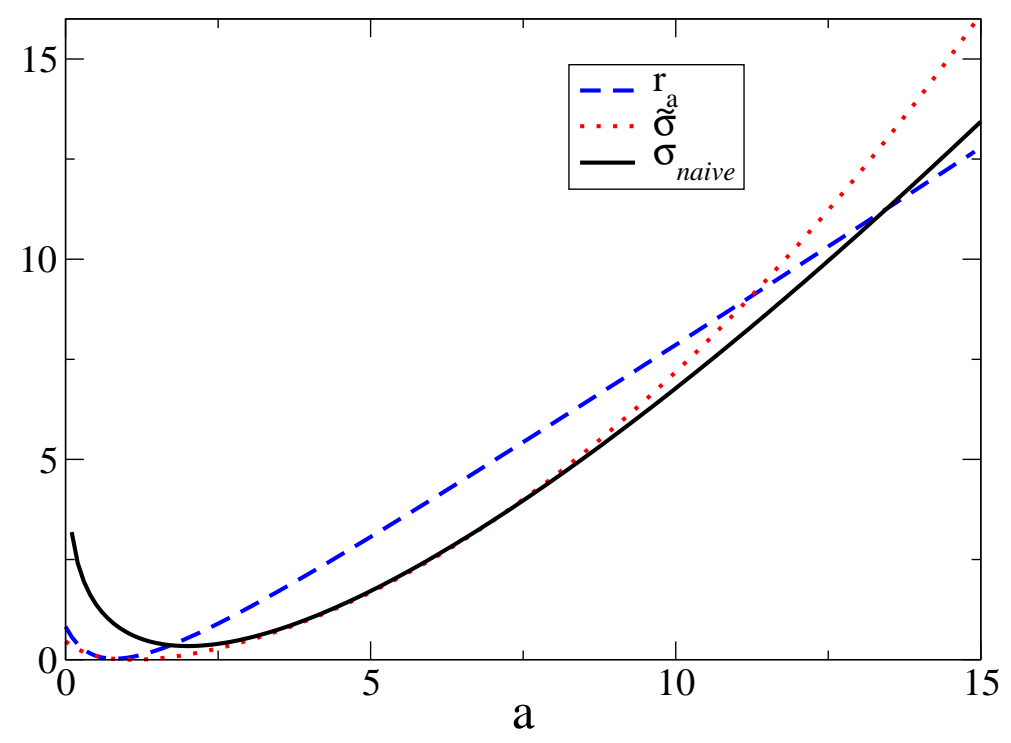

Figure 1 . The constants $r_{\mathrm{a}}, \widetilde{\sigma}$ and $\sigma_{\text {naive }}$ as functions of the parameter $a$ for the random walk on the ring in Section 4.2 with fixed parameters $b=1.7, c=0.8$ and $d=2$.

Due to Proposition $3.3 r$ is lower bounded by $r_{\mathrm{a}}$. Due to Proposition $3.4 \widetilde{\sigma}$ lower bounds $\widehat{\sigma}$ and $\sigma^{*}$. We concentrate on the comparison between the constants $r_{\mathrm{a}}, \widetilde{\sigma}$ (which are optimal in the sense clarified by Propositions 3.3 and 3.4) and $\sigma_{\text {naive. As }}$ shown in Figure 1, there is no fixed order either between $r_{\mathrm{a}}$ and $\sigma_{\text {naive }}$ or between $\widetilde{\sigma}$ and $\sigma_{\text {naive }}$. Note that, for $\tau \rightarrow 0$, the universal constant $\left(e^{\tau \sigma_{\text {naive }}}-1\right) / \tau$ converges to $\sigma_{\text {naive }}$. As a consequence there is no optimality either between the GTUR (4.5) and (GTUR 7) or between (GTUR 3) and (GTUR 7). Figure 1 shows also that there is no fixed order between $r_{a}$ and $\widetilde{\sigma}$, i.e. (GTUR 3) and (GTUR 4) are non-comparable bounds.

\section{LOCAL BOUNDS ON $I_{\alpha, \gamma}$ AND GTUR's FOR $Y_{\alpha, \gamma}^{(n)}$ : CASE OF GENERIC $\alpha$}

Our first aim is to describe a general method to get local quadratic upper bounds on $I_{\alpha, \gamma}$ around its minimum point $y_{\alpha, \gamma}$, thus leading also to lower bounds on $D_{\alpha, \gamma}$ via (2.17). This method is an extension of the one used for the empirical currents in [1, Section 4.3].

It is convenient to introduce the concept of generalized flow, which is defined as a flow without the restriction of non-negativity. In other words, we will call generalized flow any function $k: V \times V \rightarrow \mathbb{R}$ which is zero outside $E$. If $k$ is non-negative, then $k$ is a flow. The divergence of $k$ is defined as

$$
\operatorname{div}_{i} k:=\sum_{j} k_{i j}-\sum_{j} k_{j i}
$$

Due to (2.13) one has

$$
I_{\alpha, \gamma}(y) \leq I(Q, \rho), \quad \forall(Q, \rho) \in \mathcal{F}_{\alpha, \gamma, y},
$$

where the set $\mathcal{F}_{\alpha, \gamma, y}$ is defined after in (2.14). Moreover, the function $\Phi(q, p)$ defined in (2.12) satisfies the following bound obtained by a Taylor's expansion around the 
arbitrary diagonal point $(a, a)$ :

$$
\Phi(q, p)=\frac{1}{2 a}(p-q)^{2}+o\left((q-a)^{2}+(p-a)^{2}\right) .
$$

Due to (2.10), when $y$ is close to the asymptotic value $y_{\alpha, \gamma}$, it is natural to look for pairs $(Q, \rho) \in \mathcal{F}_{\alpha, \gamma, y}$ which are obtained as perturbation of $(\mathcal{Q}, \pi)$. To this aim, it is convenient to use the representation

$$
\left\{\begin{array}{l}
Q=\mathcal{Q}+\frac{y-y_{\alpha, \gamma}}{y_{\alpha, \gamma}} R \\
\rho=\pi+\frac{y-y_{\alpha, \gamma}}{y_{\alpha, \gamma}} m
\end{array}\right.
$$

We assume $y_{\alpha, \gamma} \neq 0$ in this equation. For the case $y_{\alpha, \gamma}=0$, the equation has to be modified, as explained in Remark 5.1 below.

Note that $(Q, \rho) \in \mathcal{F}_{\alpha, \gamma, y}$ if and only if the following properties are satisfied by the pair $(R, m)$ :

(P1) $R=(R(t))_{t>0}$ is a time-periodic generalized flow and therefore $R(t): V \times$ $V \rightarrow \mathbb{R}$ is zero outside $E$ for all $t \geq 0$;

(P2) $m=(m(t))_{t>0}$ is time-periodic and $m(t): V \rightarrow \mathbb{R}$ satisfies $\sum_{i} m_{i}(t)=0$ for all $t \geq 0$

(P3) $\partial_{t} m(t)+\operatorname{div} R(t)=0$,

(P4) $y_{\alpha, \gamma}=\overline{\langle\alpha, R\rangle}+\overline{\langle\gamma, m\rangle}$;

(P5) the functions in the r.h.s. of (5.4) take non-negative values.

We point out that, given $R, m$ satisfying $(\mathrm{P} 1)$ and $(\mathrm{P} 2)$, since $\mathcal{Q}_{i j}(t)>0$ for all $(i, j) \in E$ and $\pi_{i}(t)>0$ for all $i \in V$, property (P5) is satisfied for $y$ sufficiently close to $y_{\alpha, \gamma}$. Since our bounds are local for $y$ close to $y_{\alpha, \gamma}$, we will disregard (P5) in what follows.

Theorem 1. For any pair $(R, m)$ fulfilling the above properties $(P 1), \ldots,(P 4)$ the following local quadratic upper bound holds:

$$
I_{\alpha, \gamma}(y) \leq \frac{1}{2} \frac{\left(y-y_{\alpha, \gamma}\right)^{2}}{y_{\alpha, \gamma}^{2}} \sum_{(i, j) \in E} \overline{\left(\frac{\left(R_{i j}-m_{i} w_{i j}\right)^{2}}{\mathcal{Q}_{i j}}\right)}+o\left(\left(y-y_{\alpha, \gamma}\right)^{2}\right)
$$

In particular, we have the lower bound

$$
2 D_{\alpha, \gamma} \geq y_{\alpha, \gamma}^{2}\left\{\sum_{(i, j) \in E} \overline{\left(\frac{\left(R_{i j}-m_{i} w_{i j}\right)^{2}}{\mathcal{Q}_{i j}}\right)}\right\}^{-1} .
$$

We point out that, since (5.4) defines a bijection $(R, m) \mapsto(Q, \rho)$, one would get an identity in (5.5) and (5.6) by optimizing among $(R, m)$ in the above theorem. 
Proof. From (2.14) and (5.3) by setting $a=\mathcal{Q}_{i j}(t)$, we have

$$
\begin{aligned}
I(Q, \rho): & =\sum_{(i, j) \in E} \overline{\Phi\left(Q_{i j}(t), \rho_{i}(t) w_{i j}(t)\right)} \\
& =\sum_{(i, j) \in E} \overline{\left(\frac{\left(Q_{i j}-\rho_{i} w_{i j}\right)^{2}}{2 \mathcal{Q}_{i j}}+\mathcal{E}_{i j}\right)} \\
& =\frac{1}{2} \frac{\left(y-y_{\alpha, \gamma}\right)^{2}}{y_{\alpha, \gamma}^{2}} \sum_{(i, j) \in E} \overline{\left(\frac{\left(R_{i j}-m_{i} w_{i j}\right)^{2}}{\mathcal{Q}_{i j}}+\mathcal{E}_{i j}\right)},
\end{aligned}
$$

where the error term $\mathcal{E}_{i j}(t)$ is given by

$$
\mathcal{E}_{i j}(t)=o\left(\left(Q_{i j}(t)-\mathcal{Q}_{i j}(t)\right)^{2}\right)+o\left(\left(\rho_{i}(t)-\pi_{i}(t)\right)^{2}\right)=o\left(\left(y-y_{\alpha, \gamma}\right)^{2}\right) .
$$

Equations (5.7) and (5.8) imply (5.5). Finally, (5.6) follows from (5.5) by means of (2.17).

Remark 5.1. When $y_{\alpha, \gamma}=0$ the above arguments remain valid by making the following changes. Formula (5.4) becomes $Q=\mathcal{Q}+y R, \rho=\pi+y m$. In (P4) one replaces $y_{\alpha, \gamma}$ with 1 . In (P5) the $y_{\alpha, \gamma}$ 's on the numerator are 0 while the ones on the denominator become 1 . Then Theorem 11 remains valid by replacing $y_{\alpha, \gamma}$ with 1 in the denominator of (5.5) (the $y_{\alpha, \gamma}$ in the numerator is zero) and in (5.6).

Theorem 1 provides a very general method from which several local quadratic bounds and GTUR's can be derived by inserting different choices of $(R, m)$. To get sharp and interesting bounds it is important to select special perturbations $(R, m)$ fulfilling the above properties (P1)-(P4). We discuss below some special choices, leading to some corollaries of Theorem 1, This is of course not a complete list and one can find other choices in [1].

A first class of choices, closely related to the ones in [1, Section 4.5], is given in the following corollary:

Corollary 5.2. Suppose that $\mathcal{K}(t)=\left(\mathcal{K}_{i j}(t)\right)$ is a time-periodic generalized flow with $\operatorname{div} \mathcal{K}=0$ and such that $\overline{\langle\alpha, \mathcal{K}\rangle} \neq 0$. Then it holds

$$
I_{\alpha, \gamma}(y) \leq \frac{1}{4} \frac{\widehat{\sigma}}{\overline{\langle\alpha, \mathcal{K}\rangle}^{2}}\left(y-y_{\gamma, \alpha}\right)^{2}+o\left(\left(y-y_{\gamma, \alpha}\right)^{2}\right)
$$

and

$$
D_{\alpha, \gamma} \geq \frac{\overline{\langle\alpha, \mathcal{K}\rangle}^{2}}{\widehat{\sigma}}
$$

where

$$
\widehat{\sigma}:=2 \sum_{(i, j) \in E} \overline{\left(\frac{\mathcal{K}_{i j}^{2}}{\mathcal{Q}_{i j}}\right)} .
$$

Proof. It is enough to apply Theorem 1 with $R:=\left(y_{\alpha, \gamma} / \overline{\langle\alpha, \mathcal{K}\rangle}\right) \mathcal{K}$ and $m=0$.

We collect some comments on the above Corollary 5.2 .

- A possible choice of $\mathcal{K}$ is given by $\mathcal{K}=\overline{\mathcal{Q}}$ when $\langle\bar{\alpha}, \overline{\mathcal{Q}}\rangle \neq 0$. 
- When $\gamma \equiv 0$ and $\alpha$ is time-independent we have that $y_{\alpha, \gamma}=\overline{\langle\alpha, \mathcal{Q}\rangle}=\langle\alpha, \overline{\mathcal{Q}}\rangle$. In particular, by taking $\mathcal{K}=\overline{\mathcal{Q}}$ in the above Corollary [5.2, (5.10) becomes GTUR 1 valid whenever $\langle\alpha, \overline{\mathcal{Q}}\rangle \neq 0$.

- Another possible choice for $\mathcal{K}$ is given by $\mathcal{K}_{i j}(t)=\mu_{i}(t) w_{i j}(t)$, where $\mu_{i}(t)$ denotes the so-called accompanying distribution, i.e. the invariant distribution for the time-homogeneous Markov chain with time-independent rates $w_{i j}(t)$ ( $t$ thought of as frozen). For this second choice we also refer to [1, Section 4.5].

- The property of being a time periodic generalized flow with zero divergence is preserved by linear combinations. In particular, one can also take $\mathcal{K}_{i j}=$ $c_{1} \overline{\mathcal{Q}}_{i j}+c_{2} \mu_{i}(t) w_{i j}(t)$, for any fixed $c_{1}, c_{2} \in \mathbb{R}$.

- Given the model, one can look for more efficient choices of $\mathcal{K}$ by using Schnakenberg's cycle theory [7, 43] to build divergence-free flows, and afterwards by trying to optimize among these flows. Note that non-trivial divergence-free flows on the graph $(V, E)$ always exist.

We are not going to discuss in detail the possible optimization problems related to the last comment above, concerning Schnakenberg's cycle theory, since this approach is very model-dependent. We consider in the next section just one special case where an argument of this type works naturally (cf. first proof of Theorem 2).

In [27] the authors consider functionals of the form (2.9) with $\alpha$ antisymmetric and $\gamma$ not arbitrary, but of the form

$$
\gamma_{i}(t)=\frac{d}{d t} g_{i}(t) \quad \forall i \in V,
$$

for some periodic function $g_{i}$. The above form (5.12) is equivalent to the property

$$
\bar{\gamma}_{i}=0 \quad \forall i \in V .
$$

In the following result we consider general weights $\alpha$ and we weaken condition (5.13) on $\gamma$.

Corollary 5.3. Suppose that the entries of $\bar{\gamma}$ are not all strictly positive, and not all strictly negative. Fix any time-independent probability measure $p=\left(p_{i}\right)_{i \in V}$ on $V$ with $\langle p, \bar{\gamma}\rangle=0$. Recall the constant $C(p)$ defined in (3.2). Then we have the upper bound

$$
I_{\alpha, \gamma}(y) \leq \frac{C(p)}{4} \frac{\left(y-y_{\alpha, \gamma}\right)^{2}}{y_{\alpha, \gamma}^{2}}+o\left(\left(y-y_{\alpha, \gamma}\right)^{2}\right) .
$$

As a consequence we have (GTUR 2).

Proof. We take

$$
R(t):=\mathcal{Q}(t), \quad m(t):=\pi(t)-p .
$$

Properties (P1), (P2), (P3) are satisfied (recall the continuity equation (2.5)). Due to (2.10) and (5.13) we have

$$
\overline{\langle\alpha, R\rangle}+\overline{\langle\gamma, m\rangle}=y_{\alpha, \gamma}-\langle\bar{\gamma}, p\rangle=y_{\alpha, \gamma} .
$$

Hence, also property $(\mathrm{P} 4)$ is satisfied. Note that

$$
R_{i j}(t)-m_{i}(t) w_{i j}(t)=\mathcal{Q}_{i j}(t)-\left(\pi_{i}(t)-p_{i}\right) w_{i j}(t)=p_{i} w_{i j}(t) .
$$

By plugging the above identity in (5.5) and (5.6) we get (5.14). (GTUR 2) then follows due to (2.17). 
Remark 5.4. We would like to point out that the art of finding good bounds is related to the art of finding good perturbations $(m, R)$ and this is essentially the art of finding periodic solutions of the continuity equation in condition (P3). We briefly discuss in Appendix B two possible approaches.

\section{LOCAL BOUNDS ON $I_{\alpha, \gamma}$ AND GTUR's FOR $Y_{\alpha, \gamma}^{(n)}$ : CASE OF ANTISYMMETRIC $\alpha$}

In all this section we will assume, without further mention, that

$$
\left\{\begin{array}{l}
\alpha \text { is antisymmetric, i.e. } \alpha_{i j}(t)=-\alpha_{j i}(t), \\
(i, j) \in E \text { if and only if }(j, i) \in E .
\end{array}\right.
$$

Below we provide two general methods to get local quadratic bounds on $I_{\alpha, \gamma}$ (see Theorems 2 and 3) and we discuss some corollaries. We prove Theorem 2 using two approaches. For the first proof, we start with Theorem 1 and perform an optimization among flows. Hence, Theorem 2 can be seen as corollary of Theorem 11, For the second proof, we use the LD rate functional associated with the empirical current and empirical measure from [5]. Also for Theorem 3 we provide two alternative derivations. In one derivation we get Theorem 3 from Theorem 2, As a consequence, both Theorems 2 and 3 follow from Theorem 1 .

6.1. Preliminaries and Theorem 2, In what follows, we call current any function $d: V \times V \rightarrow \mathbb{R}$ which is zero outside $E$ and antisymmetric, i.e. $d_{i j}=-d_{j i} \forall i, j$. We order the elements of $V$ (arbitrarily) and write $<$ for the order relation. Given $a: V \times V \rightarrow \mathbb{R}$, we define

$$
\langle\langle a, d\rangle\rangle:=\sum_{(i, j) \in E: i<j} a_{i j} d_{i j}
$$

Note that, when also $a$ is antisymmetric, we have $\langle\langle a, d\rangle\rangle=\frac{1}{2}\langle a, d\rangle$. Finally, we define the divergence of a current $d$ by

$$
\operatorname{div}_{i} d=\sum_{j} d_{i j}
$$

We point out that the divergence of a current is defined differently from the divergence of a generalized flow (see (5.1)). This definition guarantees that, if $k$ is a generalized flow and $d$ is the current $d_{i j}:=k_{i j}-k_{j i}$, then $\left\langle\left\langle d^{\prime}, d\right\rangle\right\rangle=\left\langle d^{\prime}, k\right\rangle$ for any current $d^{\prime}$.

Due to the antisymmetry of the increments $\alpha_{i j}$, the LD rate functional $I_{\alpha, \gamma}$ admits an alternative variational characterization, in addition to (2.13), in terms of the empirical current and density [5], as explained below. We consider the function

$$
\psi(j, g, a):=\sqrt{g^{2}+a^{2}}-\sqrt{j^{2}+a^{2}}+j\left[\sinh ^{-1}(j / a)-\sinh ^{-1}(g / a)\right],
$$

$\sinh ^{-1}(x)$ denoting the hyperbolic arcsinus. Then it holds

$$
I_{\alpha, \gamma}(y)=\inf \left\{I_{*}(J, \rho):(J, \rho) \in \mathcal{F}_{\alpha, \gamma, y}^{*}\right\},
$$


where now

$$
\begin{aligned}
I_{*}(J, \rho) & :=\sum_{(i, j) \in E: i<j} \overline{\Psi\left(J_{i j}(t), G_{i j}(t), a_{i j}(t)\right)}, \\
G_{i j}(t) & :=\rho_{i}(t) w_{i j}(t)-\rho_{j}(t) w_{j i}(t), \\
a_{i j}(t) & :=2 \sqrt{\rho_{i}(t) \rho_{j}(t) w_{i j}(t) w_{j i}(t)},
\end{aligned}
$$

and $\mathcal{F}_{\alpha, \gamma, y}^{*}$ denotes the family of pairs $(J, \rho)=(J(t), \rho(t))_{t \geq 0}$ such that

(i) $J(t)$ is a time-periodic current, i.e. $J(t)=J(t+\tau)$ and $J(t)$ is an antisymmetric function on $V \times V$ which is zero outside $E$ for each time $t$;

(ii) $\rho(t)$ is a time-periodic probability measure on $V$;

(iii) the continuity equation $\partial_{t} \rho(t)+\operatorname{div} J(t)=0$ is satisfied (cf. (6.1));

(iv) $y=\overline{\langle\langle\alpha, J\rangle\rangle}+\overline{\langle\gamma, \rho\rangle}$.

According to [5, Theorem 3], formula (6.4) with the restrictions (i), (ii) and (iii) is the joint LD rate function for the empirical current and measure with speed $n \tau$. The empirical current $J^{(n)}$ is defined as the measure on $E \times[0, \tau)$ given by $J^{(n)}(i, j, d t):=Q^{(n)}(i, j, d t)-Q^{(n)}(j, i, d t)$ (cf. Section 2.2). Formula (6.4) can be deduced directly by contraction starting from the joint LD rate functional for the empirical measure and flow discussed in Section 2.2. As in (2.15) we have

$$
Y_{\alpha, \gamma}^{(n)}=\frac{1}{\tau} \sum_{(i, j) \in E: i<j} \int \alpha_{i j}(t) J^{(n)}(i, j, d t)+\frac{1}{\tau} \sum_{i} \int \gamma_{i}(t) \rho^{(n)}(i, d t),
$$

thus allowing to derive the LD principle for $Y_{\alpha, \gamma}^{(n)}$ from the LD principle of $\left(J^{(n)}, \rho^{(n)}\right)$ by contraction. We point out that the asymptotic pair $(\mathcal{J}, \pi)$ belongs to $\mathcal{F}_{\alpha, \gamma, y}^{*}$ with $y=y_{\alpha, \gamma}$ and that it fulfills the identity $I_{*}(\mathcal{J}, \pi)=0$.

As in Section 5, from now on we assume that $y_{\alpha, \gamma} \neq 0$.

Remark 6.1. Suppose that the transition graph $(V, E)$ has the property that (i) for each edge in $E$ also the reversed edge belongs to $E$, (ii) the non-oriented graph obtained from $(V, E)$ by disregarding the edge orientation is a tree. In this case, being divergence-free, $\overline{\mathcal{J}}$ must be zero and, as a consequence, $y_{\alpha, 0}=0$ for currents with time-independent increments. Moreover, reasoning as in the last paragraph of

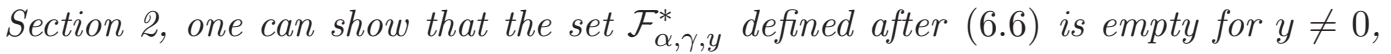
thus implying that $I_{\alpha, \gamma}(y)=+\infty$ for $y \neq 0$ and $I_{\alpha, \gamma}(0)=0$.

As in Section 5 we consider pairs $(J, \rho)$ written as perturbations of the stationary pair $(\mathcal{J}, \pi)$ as follows:

$$
\left\{\begin{array}{l}
J=\mathcal{J}+\frac{y-y_{\alpha, \gamma}}{y_{\alpha, \gamma}} Z \\
\rho=\pi+\frac{y-y_{\alpha, \gamma}}{y_{\alpha, \gamma}} m
\end{array}\right.
$$

To assure that $(J, \rho) \in \mathcal{F}_{\alpha, \gamma, y}^{*}$, the pair $(Z, m)$ must satisfy the following properties:

$\left(\mathrm{P}^{*}\right) Z=(Z(t))_{t>0}$ is a time-periodic current (in particular $Z(t): V \times V \rightarrow \mathbb{R}$ is antisymmetric and is zero outside $E$ for all $t \geq 0$ );

$\left(\mathrm{P} 2^{*}\right) m=(m(t))_{t \geq 0}$ is time-periodic and $m(t): V \rightarrow \mathbb{R}$ satisfies $\sum_{i} m_{i}(t)=0$ for all $t \geq 0$

(P3*) $\partial_{t} m+\operatorname{div} Z(t)=0$ (cf. (6.1) $)$;

$\left(\mathrm{P} 4^{*}\right) y_{\alpha, \gamma}=\overline{\langle\langle\alpha, Z\rangle\rangle}+\overline{\langle\gamma, m\rangle}$; 
$\left(\mathrm{P}^{*}\right)$ it holds $\pi_{i}(t)+\frac{y-y_{\alpha, \gamma}}{y_{\alpha, \gamma}} m_{i}(t) \geq 0$ for all $t \geq 0$ and $i \in V$.

Since $\pi_{i}(t)>0$ for any $t$, condition $\left(\mathrm{P} 5^{*}\right)$ is satisfied for $y$ near enough to $y_{\alpha, \gamma}$. As a consequence, in what follows we disregard condition $\left(\mathrm{P} 5^{*}\right)$.

We can finally state our general method to get local quadratic bounds on $I_{\alpha, \gamma}$ :

Theorem 2. For any pair $(Z, m)$ fulfilling the above properties $\left(P 1^{*}\right), \ldots,\left(P 4^{*}\right)$ the following local quadratic upper bound holds:

$$
I_{\alpha, \gamma}(y) \leq \frac{1}{2} \frac{\left(y-y_{\alpha, \gamma}\right)^{2}}{y_{\alpha, \gamma}^{2}} \sum_{(i, j) \in E: i<j} \overline{\left(\frac{\left(Z_{i j}-\left(m_{i} w_{i j}-m_{j} w_{j i}\right)\right)^{2}}{\mathcal{Q}_{i j}+\mathcal{Q}_{j i}}\right)}+o\left(\left(y-y_{\alpha, \gamma}\right)^{2}\right) .
$$

In particular, we have the lower bound

$$
2 D_{\alpha, \gamma} \geq y_{\alpha, \gamma}^{2}\left\{\sum_{(i, j) \in E: i<j} \overline{\left(\frac{\left(Z_{i j}-\left(m_{i} w_{i j}-m_{j} w_{j i}\right)\right)^{2}}{\mathcal{Q}_{i j}+\mathcal{Q}_{j i}}\right)}\right\}^{-1} .
$$

We point out that, since (6.8) defines a bijection $(Z, m) \mapsto(J, \rho)$, one would get an identity in (6.9) and (6.10) by optimizing among $(Z, m)$ in the above theorem.

6.2. First proof of Theorem 2 , The proof relies on Theorem 1 and an optimization procedure in the same spirit of the last comment on Corollary 5.2.

Let $(Z, m)$ be a pair fulfilling properties $\left(\mathrm{P} 1^{*}\right), \ldots,\left(\mathrm{P} 4^{*}\right)$ and let $R^{\prime}$ be the timeperiodic generalized flow given by $R_{i j}^{\prime}(t):=Z_{i j}(t) / 2$. Note that the pair $\left(R^{\prime}, m\right)$ satisfies properties $(\mathrm{P} 1), \ldots,(\mathrm{P} 4)$ in Section 5 , We take $R=(R(t))_{t \geq 0}$ as $R(t):=$ $R^{\prime}(t)+S(t)$, where $S(t)$ is a generic time-periodic symmetric generalized flow, i.e. $S_{i, j}(t)=S_{j, i}(t)$ for all $i, j, t$. Since $\operatorname{div} S(t)=0$ and $\langle\alpha(t), S(t)\rangle=0$ by the antisymmetry of $\alpha$, also the pair $(R, m)$ satisfies conditions $(\mathrm{P} 1), . .,(\mathrm{P} 4)$ and therefore Theorem 1 applies to $(R, m)$.

We optimize the upper bound (5.5) in Theorem 1 over the symmetric generalized flows $S$. For the optimization, the basic computation that we need is the following. We consider some fixed numbers $r_{k}, a_{k}, q_{k}, k=1,2$ and compute

$$
\inf _{s \in \mathbb{R}}\left[\frac{\left(r_{1}+s-a_{1}\right)^{2}}{q_{1}}+\frac{\left(r_{2}+s-a_{2}\right)^{2}}{q_{2}}\right] .
$$

The function is minimized at

$$
s^{*}=\frac{c_{1}}{c_{1}+c_{2}}\left(a_{1}-r_{1}\right)+\frac{c_{2}}{c_{1}+c_{2}}\left(a_{2}-r_{2}\right),
$$

where $c_{k}:=q_{k}^{-1}$. The minimal value is given by

$$
\frac{\left[\left(r_{1}-r_{2}\right)-\left(a_{1}-a_{2}\right)\right]^{2}}{q_{1}+q_{2}} .
$$

Let us come back to the upper bound (5.5) in Theorem 11. Independently for each pair of edges $(i, j)$ and $(j, i)$, we can evaluate

$$
\inf _{s \in \mathbb{R}}\left\{\frac{\left(R_{i j}^{\prime}(t)+s-m_{i}(t) w_{i j}(t)\right)^{2}}{\mathcal{Q}_{i j}(t)}+\frac{\left(R_{j i}^{\prime}(t)+s-m_{j}(t) w_{j i}(t)\right)^{2}}{\mathcal{Q}_{j i}(t)}\right\},
$$


where $s$ has to be thought as the value $S_{i j}(t)=S_{j i}(t)$. According to (6.12) the above infimum is indeed attained at a suitable value $S_{i j}^{*}(t)$ and equals

$$
\frac{\left[\left(R_{i j}^{\prime}(t)-R_{j i}^{\prime}(t)\right)-\left(m_{i}(t) w_{i j}(t)-m_{j}(t) w_{j i}(t)\right)\right]^{2}}{\mathcal{Q}_{i j}(t)+\mathcal{Q}_{j i}(t)} .
$$

As a consequence, by taking $R(t)=R^{\prime}(t)+S^{*}$ the resulting bound (5.5) reduces to (6.9) since $Z_{i j}(t):=R_{i j}^{\prime}(t)-R_{j i}^{\prime}(t)$. Finally, (6.10) follows from (6.9) and (2.17).

6.3. Second proof of Theorem 2 . We follow the same arguments of Theorem 1 but applied to the functional (6.4). We first consider the Taylor's expansion up to the second order of the function $\Psi(j, g, a)$ around the point $(x-y, x-y, 2 \sqrt{x y})$ with $x, y \geq 0$. By writing

$$
\left\{\begin{array}{l}
j=x-y+\delta j, \\
g=x-y+\delta g, \\
a=2 \sqrt{x y}+\delta a,
\end{array}\right.
$$

we have (after cumbersome but straightforward computations) that

$$
\begin{aligned}
\psi(j, g, a) & =\frac{1}{2} \frac{1}{x+y}(j-g)^{2}+o\left((\delta j)^{2}\right)+o\left((\delta g)^{2}\right)+o\left((\delta a)^{2}\right) \\
& =\frac{1}{2} \frac{1}{x+y}(\delta j-\delta g)^{2}+o\left((\delta j)^{2}\right)+o\left((\delta g)^{2}\right)+o\left((\delta a)^{2}\right) .
\end{aligned}
$$

By (6.5) and (6.6) we can write

$$
\begin{aligned}
J_{i j}(t) & =\mathcal{Q}_{i j}(t)-\mathcal{Q}_{j i}(t)+\frac{y-y_{\alpha, \gamma}}{y_{\alpha, \gamma}} Z_{i j}(t), \\
G_{i j}(t) & =\mathcal{Q}_{i j}(t)-\mathcal{Q}_{j i}(t)+\frac{y-y_{\alpha, \gamma}}{y_{\alpha, \gamma}}\left[m_{i}(t) w_{i j}(t)-m_{j}(t) w_{j i}(t)\right], \\
a_{i j}(t) & =2 \sqrt{\mathcal{Q}_{i j}(t) \mathcal{Q}_{j i}(t)}+\delta a_{i j}(t)
\end{aligned}
$$

where $\delta a_{i j}(t)=O\left(\left|y-y_{\alpha, \gamma}\right|\right)$ (i.e. $\left|\delta a_{i j}(t)\right| \leq C\left|y-y_{\alpha, \gamma}\right|$ for $y$ near to $\left.y_{\alpha, \gamma}\right)$. Due to the above identities, applying (6.15) with $x=\mathcal{Q}_{i j}(t)$ and $y=\mathcal{Q}_{j i}(t)$ we get

$$
\begin{aligned}
& \psi\left(J_{i j}(t), G_{i j}(t), a_{i j}(t)\right)= \\
& \quad \frac{1}{2} \frac{\left(y-y_{\alpha, \gamma}\right)^{2}}{y_{\alpha, \gamma}^{2}} \frac{\left[Z_{i j}(t)-\left(m_{i}(t) w_{i j}(t)-m_{j}(t) w_{j i}(t)\right)\right]^{2}}{\mathcal{Q}_{i j}(t)+\mathcal{Q}_{j i}(t)}+o\left(\left(y-y_{\alpha, \gamma}\right)^{2}\right) .
\end{aligned}
$$

From this equation together with (6.3) and (6.4) we get (6.9). Finally, (6.10) follows from (6.9) by (2.17).

6.4. Corollaries to Theorem 2. Likewise the previous section we have also the following results.

Corollary 6.2. Suppose that $\mathcal{K}(t)=\left(\mathcal{K}_{i j}(t)\right)$ is a time-periodic current with $\operatorname{div} \mathcal{K}=$ 0 and such that $\overline{\langle\langle\alpha, \mathcal{K}\rangle\rangle} \neq 0$. Then it holds

$$
I_{\alpha, \gamma}(y) \leq \frac{1}{4} \frac{\widetilde{\sigma}}{\overline{\langle\langle\alpha, \mathcal{K}\rangle\rangle}^{2}}\left(y-y_{\gamma, \alpha}\right)^{2}+o\left(\left(y-y_{\gamma, \alpha}\right)^{2}\right)
$$


and

$$
D_{\alpha, \gamma} \geq \frac{\overline{\langle\langle\alpha, \mathcal{K}\rangle\rangle}^{2}}{\widetilde{\sigma}}
$$

where

$$
\tilde{\sigma}:=2 \sum_{(i, j) \in E: i<j} \overline{\frac{\mathcal{K}_{i j}^{2}}{\mathcal{Q}_{i j}+\mathcal{Q}_{j i}}} .
$$

We point out that Corollary 6.2 was also obtained in [1] and it is an immediate consequence of Theorem 2 with $Z:=\left(y_{\alpha, \gamma} / \overline{\langle\langle\alpha, \mathcal{K}\rangle\rangle}\right) \mathcal{K}$ and $m=0$.

As in Section 5 we can collect some comments on the above Corollary 6.2 ,

- A possible choice of $\mathcal{K}$ is given by $\mathcal{K}=\overline{\mathcal{J}}$ when $\langle\langle\bar{\alpha}, \overline{\mathcal{J}}\rangle\rangle \neq 0$.

- When $\gamma \equiv 0$ and $\alpha$ is time-independent we have that $y_{\alpha, \gamma}=\overline{\langle\langle\alpha, \mathcal{J}\rangle\rangle}=$ $\langle\langle\alpha, \overline{\mathcal{J}}\rangle\rangle$. In particular, by taking $\mathcal{K}=\overline{\mathcal{J}}$ in the above Corollary 6.2, (6.21) becomes (GTUR 3) valid whenever $\langle\alpha, \overline{\mathcal{J}}\rangle \neq 0$.

- Another possible choice for $\mathcal{K}$ is given by $\mathcal{K}_{i j}(t)=\mu_{i}(t) w_{i j}(t)-\mu_{j} w_{j i}(t)$, where $\mu_{i}(t)$ denotes the so-called accompanying distribution (cf. Section 5). For this second choice we also refer to [1, Section 4.5].

- The property of being a time periodic current with zero divergence is preserved by linear combinations. In particular, one can also take $\mathcal{K}_{i j}=$ $c_{1} \overline{\mathcal{J}}_{i j}+c_{2}\left(\mu_{i}(t) w_{i j}(t)-\mu_{j}(t) w_{j i}(t)\right)$, for any fixed $c_{1}, c_{2} \in \mathbb{R}$ (see [1, Section 4.5] for further discussions).

- Given the model, one can look for more efficient choices of $\mathcal{K}$ by using Schnakenberg's cycle theory [7, 43] to build divergence-free currents, and afterwards by trying to optimize among these currents. We recall that any divergence-free current $\mathcal{K}$ must be zero if the graph $(V, E)$ is a tree after replacing pairs of oriented edges $(i, j)$ and $(j, i)$ by the unoriented edge $\{i, j\}$. In this case Corollary 6.2 becomes empty.

Corollary 6.3. Suppose that the entries of $\bar{\gamma}$ are not all strictly positive, and not all strictly negative. Fix any time-independent probability measure $p=\left(p_{i}\right)_{i \in V}$ on $V$ with $\langle p, \bar{\gamma}\rangle=0$. Recall the definition of $C_{\mathrm{a}}(p)$ in (3.4). Then we have the upper bound

$$
I_{\alpha, \gamma}(y) \leq \frac{1}{4} \frac{\left(y-y_{\alpha, \gamma}\right)^{2}}{y_{\alpha, \gamma}^{2}} C_{\mathrm{a}}(p)+o\left(\left(y-y_{\alpha, \gamma}\right)^{2}\right) .
$$

As a consequence we have (GTUR 4).

The above result follows from Theorem 2 by taking $Z(t):=\mathcal{J}(t)$ and $m(t):=$ $\pi(t)-p$.

Remark 6.4. Note that both Corollary 6.2 and 6.3 could be derived respectively from Corollary 5.2 and 5.3 by an optimization over symmetric generalized flows as in the first proof of Theorem Q In particular, in the case of an antisymmetric $\alpha$, the bounds discussed in this section are better than the corresponding ones discussed in the previous section, since they are obtained by an optimization procedure. We recall that we have proved in a direct way this issue (cf. Prop. 3.3 and its proof in Appendix A). 
6.5. Theorem 3 and its corollaries. In Theorem 3 below we present another general method to produce quadratic bounds on the LD rate functional $I_{\alpha, \gamma}$. We provide two simple derivations of this theorem. The first one is inspired by the approach followed in [1, Section 4.1]. The second one, based on Theorem 2, shows indeed that the bounds provided by Theorem 2 are better than the ones provided by Theorem 3 (see Remark 6.5 below). Nevertheless, the interest to Theorem 3 comes from the fact that it allows (see the corollaries below) to get GTUR's with constants resembling in their form to the average entropy production rate $\sigma$.

Theorem 3. For any pair $(Z, m)$ fulfilling properties $\left(P 1^{*}\right), \ldots,\left(P 4^{*}\right)$ the following local quadratic upper bound holds:

$I_{\alpha, \gamma}(y) \leq \frac{1}{4} \frac{\left(y-y_{\alpha, \gamma}\right)^{2}}{y_{\alpha, \gamma}^{2}} \sum_{(i, j) \in E: i<j} \overline{\left(\frac{\left(Z_{i j}-\left(m_{i} w_{i j}-m_{j} w_{j i}\right)\right)^{2}}{\mathcal{J}_{i j}} \ln \frac{\mathcal{Q}_{i j}}{\mathcal{Q}_{j i}}\right)}+o\left(\left(y-y_{\alpha, \gamma}\right)^{2}\right)$.

In particular, we have the lower bound

$$
D_{\alpha, \gamma} \geq y_{\alpha, \gamma}^{2}\left\{\sum_{(i, j) \in E: i<j} \overline{\left(\frac{\left(Z_{i j}-\left(m_{i} w_{i j}-m_{j} w_{j i}\right)\right)^{2}}{\mathcal{J}_{i j}} \ln \frac{\mathcal{Q}_{i j}}{\mathcal{Q}_{j i}}\right)}\right\}^{-1} .
$$

First proof. We have (recall (6.3) and (6.5))

$$
I_{\alpha, \gamma}(y) \leq I_{*}(J, \rho) \leq \frac{1}{4} \sum_{(i, j) \in E: i<j} \frac{1}{\tau} \int_{0}^{\tau} \frac{\left[J_{i j}(t)-G_{i j}(t)\right]^{2}}{G_{i j}(t)} \ln \frac{\rho_{i}(t) w_{i j}(t)}{\rho_{j}(t) w_{j i}(t)} d t,
$$

for any pair $(J, \rho)$ in $\mathcal{F}_{\alpha, \gamma, y}^{*}$. The second bound in (6.26) follows from Eq. (12) in [21, implying that

$$
\Psi\left(J_{i j}(t), G_{i j}(t), a_{i j}(t)\right) \leq \frac{1}{4} \frac{\left[J_{i j}(t)-G_{i j}(t)\right]^{2}}{G_{i j}(t)} \ln \frac{\rho_{i}(t) w_{i j}(t)}{\rho_{j}(t) w_{j i}(t)} .
$$

We take the pair $(J, \rho)$ as in (6.8). Then, for $y$ close to $y_{\alpha, \gamma}$, we have that $(J, \rho) \in$ $\mathcal{F}_{\alpha, \gamma, y}^{*}$ and therefore we can apply (6.26) to $(J, \rho)$. The thesis then follows by a Taylor's expansion of the r.h.s. of (6.26) for $y$ close to $y_{\alpha, \gamma}$, since

$$
\begin{aligned}
& J_{i j}(t)-G_{i j}(t)=\frac{y-y_{\alpha, \gamma}}{y_{\alpha, \gamma}}\left(Z_{i j}(t)-\left[m_{i}(t) w_{i j}(t)-m_{j}(t) w_{j i}(t)\right]\right), \\
& G_{i j}(t)=\mathcal{J}_{i j}(t)+o(1), \\
& \frac{\rho_{i}(t) w_{i j}(t)}{\rho_{j}(t) w_{j i}(t)}=\frac{\mathcal{Q}_{i j}(t)}{\mathcal{Q}_{j i}(t)}+o(1) .
\end{aligned}
$$

Second proof. The bound (6.24) is an immediate consequence of the bound (6.9) in Theorem 2 and the general inequality (cf. [1, Eq. (29)])

$$
(x-y) \ln \frac{x}{y} \geq \frac{2(x-y)^{2}}{x+y}, \quad x, y>0 .
$$

Indeed, from the above inequality one gets that $\left(\mathcal{J}_{i j}\right)^{-1} \ln \left(\mathcal{Q}_{i j} / \mathcal{Q}_{j i}\right) \geq 2\left(\mathcal{Q}_{i j}+\mathcal{Q}_{j i}\right)^{-1}$. Finally, (6.25) follows from (6.24) and (2.17). 
Remark 6.5. Due to (6.30), the r.h.s. of (6.10) in Theorem 2 is lower bounded by the r.h.s. of (6.25) in Theorem 3 . In particular, the bounds obtained by Theorem 0 are better than the corresponding bounds obtained by Theorem 3 .

Corollary 6.6. Suppose that $\mathcal{K}(t)=\left(\mathcal{K}_{i j}(t)\right)$ is a time-periodic current with $\operatorname{div} \mathcal{K}=$ 0 and such that $\overline{\langle\langle\alpha, \mathcal{K}\rangle\rangle} \neq 0$. Then it holds

$$
I_{\alpha, \gamma}(y) \leq \frac{1}{4} \frac{\sigma^{*}}{\langle\langle\langle\alpha, \mathcal{K}\rangle\rangle}{ }^{2}\left(y-y_{\gamma, \alpha}\right)^{2}
$$

and

where

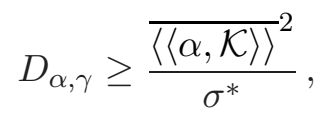

$$
\sigma^{*}:=\sum_{(i, j) \in E: i<j} \overline{\left(\frac{\mathcal{K}_{i j}^{2}}{\mathcal{J}_{i j}} \ln \frac{\mathcal{Q}_{i j}}{\mathcal{Q}_{j i}}\right)} .
$$

Proof. We apply Theorem 3 with a slight improvement, by taking $m:=0$ and $Z:=\left(y_{\alpha, \gamma} / \overline{\langle\langle\alpha, \mathcal{K}\rangle\rangle}\right) \mathcal{K}$. Theorem 3 would imply the thesis, with the exception that the bound (6.31) would be only local. On the other hand, since $m=0$, the error terms $o(1)$ in (6.28) and (6.29) are simply zero and the first proof of Theorem 3 gives that the local bound (6.24) is in this case a global bound.

We point out that Corollary 6.6 was also obtained in [1. Moreover, we observe that (GTUR 5) follows from Corollary 6.6 by taking $\mathcal{K}:=\overline{\mathcal{J}}$. Finally, by Remark 6.5 we also get that $\sigma^{*} \geq \widetilde{\sigma}$, where the constant $\widetilde{\sigma}$ is defined as in (6.22).

Corollary 6.7. Suppose that the entries of $\bar{\gamma}$ are not all strictly positive, and not all strictly negative. Fix any time-independent probability measure $p=\left(p_{i}\right)_{i \in V}$ on $V$ with $\langle p, \bar{\gamma}\rangle=0$. Recall the constant $C_{\mathrm{a}}^{*}(p)$ defined in (3.6). Then we have the upper bound

$$
I_{\alpha, \gamma}(y) \leq \frac{1}{4} \frac{\left(y-y_{\alpha, \gamma}\right)^{2}}{y_{\alpha, \gamma}^{2}} C_{\mathrm{a}}^{*}(p)+o\left(\left(y-y_{\alpha, \gamma}\right)^{2}\right) .
$$

As a consequence we have (GTUR 6).

The above corollary follows from Theorem 3 by taking $Z(t):=\mathcal{J}(t)$ and $m(t):=$ $\pi(t)-p$, as in Corollary 6.3. GTUR 6) corresponds to [27, Eq. (14)]. We point out that, by Remark 6.5, we get that $C_{\mathrm{a}}^{*}(p) \geq C_{\mathrm{a}}(p)$, where the constant $C_{\mathrm{a}}(p)$ is defined as in (3.4).

\section{GTUR 7) AND ITS EXTENSIONS}

In this section we generalize the results from [42] to general protocols that can be time-asymmetric. Our GTUR contains the rate $\sigma_{\text {naive }}$ that becomes the average entropy production rate $\sigma$ for the case of time-symmetric protocols, as explained in Section 3 .

We denote by $\Theta_{\tau}$ the set of all possible paths of the Markov chain up to time $\tau$ $\left(\Theta_{\tau}\right.$ is given by the piecewise-constant paths $\left.\Gamma:[0, \tau] \rightarrow V\right)$. Note that $\tau$ is both the period and the length of the paths. We write $\mathcal{R}_{\tau}: \Theta_{\tau} \rightarrow \Theta_{\tau}$ for the time-reflection around $\tau / 2$ and we denote by $P$ the probability measure on $\Theta_{\tau}$ given by the law of the random path $(X(t))_{0 \leq t \leq \tau}$ when the Markov chain has initial distribution $\pi(0)$. 
Similarly to [5] we introduce the average entropy flow from naive reversal defined as the entropy $H\left[P \mid P \circ \mathcal{R}_{\tau}\right]$ of $P$ w.r.t. $P \circ \mathcal{R}_{\tau}$ (note that $\mathcal{R}_{\tau}=\mathcal{R}_{\tau}^{-1}$ ), i.e.

$$
H\left[P \mid P \circ \mathcal{R}_{\tau}\right]=\int_{\Theta_{\tau}} P(d \Gamma) \ln \frac{d P}{d\left(P \circ R_{\tau}\right)}(\Gamma),
$$

where $P \circ \mathcal{R}_{\tau}(A):=P\left(\mathcal{R}_{\tau}(A)\right)$. One gets (cf. [5, Section 4])

$$
H\left[P \mid P \circ \mathcal{R}_{\tau}\right]=\tau \sigma_{\text {naive }},
$$

where $\sigma_{\text {naive }}$ is given by (3.7).

Given a function $F: \Theta_{\tau} \rightarrow \mathbb{R}$, we define the empirical functional $Y_{F}^{(n)}$ as

$$
Y_{F}^{(n)}:=\frac{1}{n} \sum_{j=0}^{n-1} F\left(\left(X_{j \tau+s}\right)_{0 \leq s \leq \tau}\right) .
$$

We point out that the empirical functional $Y_{\alpha, \gamma}^{(n)}$ given in (2.9) can be written as $Y_{\alpha, \gamma}^{(n)}=Y_{F}^{(n)}$ by defining $F$ as

$$
F(\Gamma):=\frac{1}{\tau} \sum_{\substack{t \in(0, \tau]: \\ \Gamma(t-) \neq \Gamma(t+)}} \alpha_{\Gamma(t-), \Gamma(t+)}(t)+\frac{1}{\tau} \int_{0}^{\tau} \gamma_{\Gamma(t)}(t) d t .
$$

We remark that $Y_{\alpha, \gamma}^{(n)}$ is a linear functional of the empirical flow and density, while the empirical functional $Y_{F}^{(n)}$ in (7.3) is more general.

Theorem 4. [42] revisited] Let $F: \Theta_{\tau} \rightarrow \mathbb{R}$ be antisymmetric, i.e. $F=-F \circ \mathcal{R}_{\tau}$. Then, as $n \rightarrow \infty, Y_{F}^{(n)}$ satisfies an LDP with speed $n$. Calling $I_{F}$ the associated LD rate function, calling $y_{F}$ the asymptotic value of $Y_{F}^{(n)}$ and assuming $y_{F} \neq 0$, it holds

$$
I_{F}^{\prime \prime}\left(y_{F}\right) \leq \frac{1}{2 y_{F}^{2}}\left(e^{\tau \sigma_{\text {naive }}}-1\right) .
$$

As a consequence, one has the GTUR

$$
\frac{D_{F}}{y_{F}^{2}} \geq \frac{1}{e^{\tau \sigma_{\text {naive }}}-1}
$$

where $D_{F}$ is the asymptotic diffusion coefficient given by

$$
2 D_{F}:=\lim _{n \rightarrow \infty} n \operatorname{Var}\left(Y_{F}^{(n)}\right) .
$$

We note that $y_{F}=E[F]$ and $2 D_{F}=\operatorname{Var}(F)$, where the expectation and the variance are computed w.r.t. $P$.

We stress that Theorem 4 holds for any protocol, but it is restricted to antisymmetric functionals $F$ as in [42]. In Appendix $C$ we give for completeness the derivation of Theorem 4. This proof follows the main steps of the one in [42], while some mathematical structures are investigated more carefully.

In order to apply Theorem 4 to the functional $Y_{\alpha, \gamma}^{(n)}=Y_{F}^{(n)}$, with $F$ defined in (7.4), we need that $F$ is antisymmetric and this holds whenever

$$
\left\{\begin{array}{l}
\alpha_{i, j}(t)=-\alpha_{j, i}(\tau-t) \\
\gamma_{i}(t)=-\gamma_{i}(\tau-t)
\end{array}\right.
$$


for all $i, j \in V$ and all $t \in[0, \tau]$. If the weights are time independent, then (7.8) reduces to the fact that $\alpha$ is antisymmetric (i.e. $\alpha_{i, j}=\alpha_{j, i}$ ) and $\gamma \equiv 0$. Let us finally explain how to get (GTUR 7). By (2.16) and (7.7) we have

$$
2 D_{\alpha, \gamma}=\lim _{n \rightarrow \infty} n \tau \operatorname{Var}\left(Y_{\alpha, \gamma}^{(n)}\right)=\lim _{n \rightarrow \infty} n \tau \operatorname{Var}\left(Y_{F}^{(n)}\right)=2 \tau D_{F},
$$

while

$$
y_{\alpha, \gamma}=y_{F} .
$$

As a consequence, we get that $D_{\alpha, \gamma} / y_{\alpha, \gamma}^{2}=\tau D_{F} / y_{F}^{2}$. As a byproduct with (7.6), we get the desired (GTUR 7).

\section{Appendix A. Proof of Propositions 3.1, 3.3, 3.4 and Remark 3.2}

A.1. Proof of Proposition 3.1. The universal rate in (GTUR 2) can be written as $C(p)=\sum_{i} p_{i}^{2} X_{i}$, where $X_{i}=1 / A_{i}, A_{i}$ is defined in (3.8), and $A_{i}>0$. By (GTUR 2) we have that

$$
\frac{D_{\alpha, \gamma}}{y_{\alpha, \gamma}^{2}} \geq \frac{1}{C_{\star}}
$$

where $C_{\star}$ is the infimum of $C(p)$ as $p=\left(p_{i}\right)_{i \in V}$ varies among the probability measures on $V$ with $\langle\bar{\gamma}, p\rangle=0$. Below we show that the convex function $p \mapsto C(p)$, defined on the set of probability measures with $\langle\bar{\gamma}, p\rangle=0$, has exactly one extremal point, hence this extremal point must be the minimum point.

By the Lagrange's multipliers method, we look to the extremal points of the function

$$
f(p)=\sum_{i} p_{i}^{2} X_{i}-a\left(\sum_{i} p_{i}-1\right)-b\left(\sum_{i} p_{i} \bar{\gamma}_{i}\right)
$$

$a, b$ being the multipliers. The extremal point satisfies $2 p_{i}^{\star} X_{i}-a-b \bar{\gamma}_{i}=0$ for all $i \in V$, i.e.

$$
p_{i}^{\star}=\frac{a+b \bar{\gamma}_{i}}{2 X_{i}}=\frac{a A_{i}+b A_{i} \bar{\gamma}_{i}}{2} \quad \forall i \in V .
$$

The constants $a, b$ are fixed by imposing that $\sum_{i} p_{i}^{\star}=1$ and $\left\langle\bar{\gamma}, p^{\star}\right\rangle=0$. This is equivalent to the system

$$
\left\{\begin{array}{l}
a A+b B=2 \\
a B+b C=0
\end{array}\right.
$$

with $A:=\sum_{i} A_{i}, B:=\sum_{i} A_{i} \bar{\gamma}_{i}$ and $C:=\sum_{i} A_{i} \bar{\gamma}_{i}^{2}$.

We point out that by Cauchy-Schwarz inequality we have

$$
B^{2}=\left(\sum_{i} A_{i} \bar{\gamma}_{i}\right)^{2}=\left(\sum_{i} \sqrt{A_{i}}\left(\sqrt{A_{i}} \bar{\gamma}_{i}\right)\right)^{2} \leq\left(\sum_{i} A_{i}\right)\left(\sum_{i} A_{i} \bar{\gamma}_{i}^{2}\right)=A C .
$$

Moreover, the above bound becomes an identity if and only if the vectors $\left(A_{i}\right)$ and $\left(\sqrt{A_{i}} \bar{\gamma}_{i}\right)$ are proportional. This condition is fulfilled in the case given by Item (i) in Proposition 3.1 since $\bar{\gamma}=0$, but not in the case given by Item (ii) in Proposition 3.1. since $A_{i}>0$ for all $i$ while $\bar{\gamma} \neq 0$ has neither all entries negative nor all entries positive. Hence, for Item (ii) we have $A C \neq B^{2}$. 
If $A C \neq B^{2}$, then the solution of the system is given by $a=\frac{2 C}{A C-B^{2}}$ and $b=$ $\frac{-2 B}{A C-B^{2}}$, thus leading to

$$
\begin{aligned}
C_{\star}=C\left(p^{\star}\right)=\sum_{i}\left(p_{i}^{\star}\right)^{2} X_{i} & =\sum_{i} p_{i}^{\star}\left(p_{i}^{\star} X_{i}\right)=\sum_{i} p_{i}^{\star}\left(\frac{a+b \bar{\gamma}_{i}}{2}\right) \\
& =\frac{a}{2}+\frac{b}{2}\left(\sum_{i} p_{i}^{\star} \bar{\gamma}_{i}\right)=\frac{a}{2}+\frac{b}{2} \sum_{i} \bar{\gamma}_{i}\left(\frac{a A_{i}+b A_{i} \bar{\gamma}_{i}}{2}\right) \\
& =\frac{a}{2}+\frac{a b}{4} B+\frac{b^{2}}{4} C=\frac{C}{A C-B^{2}} .
\end{aligned}
$$

This concludes the proof of Item (ii) in Proposition 3.1 by (A.1) and by the above observation that $A C-B^{2}>0$.

For the case corresponding to Item (i) of Proposition 3.1 with $\bar{\gamma}=0$, the multiplier $b$ can be neglected and $a A=2$. Hence $p_{i}^{\star}=A_{i} / \sum_{j} A_{j}$, which leads to the identity

$$
C_{\star}=C\left(p^{\star}\right)=\sum_{i}\left(p_{i}^{*}\right)^{2} X_{i}=\left[\sum_{i} A_{i}\right]^{-1},
$$

A.2. Proof of Remark 3.2. Since $\pi_{i}$ is time-independent, the statement in Remark 3.2 is equivalent to the inequality

$$
2 \sum_{i} A_{i}=\sum_{i} \pi_{i}\left[\sum_{j:(i, j) \in E} \bar{w}_{i j}\right]^{-1} \geq\left[\sum_{(i, j) \in E}\left(\overline{\mathcal{Q}}_{i j}\right)^{2} \overline{\left(1 / \mathcal{Q}_{i j}\right)}\right]^{-1}=2 / \widehat{\sigma} .
$$

Recall that, given a positive random variable $Y$, it holds $\mathbb{E}[1 / Y] \geq 1 / \mathbb{E}[Y]$ by Jensen's inequality. We apply this inequality twice. As a first application we get $\overline{\left(1 / \mathcal{Q}_{i j}\right)} \geq 1 / \overline{\mathcal{Q}}_{i j}$. This implies that

$$
\left.\left[\sum_{(i, j) \in E}\left(\overline{\mathcal{Q}}_{i j}\right)^{2} \overline{\left(1 / \mathcal{Q}_{i j}\right)}\right]^{-1} \leq\left[\sum_{(i, j) \in E} \overline{\mathcal{Q}}_{i j}\right)\right]^{-1} .
$$

As a second application we get

$$
\left.\sum_{i} \pi_{i}\left[\sum_{j:(i, j) \in E} \bar{w}_{i j}\right]^{-1} \geq\left[\sum_{i} \pi_{i} \sum_{j:(i, j) \in E} \bar{w}_{i j}\right]^{-1}=\left[\sum_{(i, j) \in E} \overline{\mathcal{Q}}_{i j}\right)\right]^{-1} .
$$

(A.2) is then a byproduct of (A.3) and A.4 .

A.3. Proof of Proposition 3.3. The last statement in Proposition 3.3 is an immediate consequence of the bounds $C(p) \geq C_{a}(p)$ and $C_{a}^{*}(p) \geq C_{a}(p)$, on which we focus. The bound $C_{a}^{*}(p) \geq C_{a}(p)$ follows from Remark 6.5 as discussed after Corollary 6.7. Let us prove that $C(p) \geq C_{a}(p)$. Given $x, y \geq 0$ and $X, Y>0$, we have

The above bound implies

$$
\frac{(x-y)^{2}}{X+Y} \leq \frac{x^{2}+y^{2}}{X+Y}=\frac{x^{2}}{X+Y}+\frac{y^{2}}{X+Y} \leq \frac{x^{2}}{X}+\frac{y^{2}}{Y} .
$$

$$
\begin{aligned}
\sum_{(i, j) \in E} \frac{\left(p_{i} w_{i j}(t)-p_{j} w_{j i}(t)\right)^{2}}{\mathcal{Q}_{i j}(t)+\mathcal{Q}_{j i}(t)} & \leq \sum_{(i, j) \in E}\left[\frac{\left(p_{i} w_{i j}(t)\right)^{2}}{\mathcal{Q}_{i j}(t)}+\frac{\left(p_{j} w_{j i}(t)\right)^{2}}{\mathcal{Q}_{j i}(t)}\right] \\
& =2 \sum_{(i, j) \in E} \frac{\left(p_{i} w_{i j}(t)\right)^{2}}{\mathcal{Q}_{i j}(t)}
\end{aligned}
$$


By taking the time average on $[0, \tau]$ in $\left(\underline{A .5}\right.$, we conclude that $C_{a}(p) \leq C(p)$.

A.4. Proof of Proposition 3.4. The bound $\sigma^{*} \geq \widetilde{\sigma}$ has been derived in [1] and follows also from Remark 6.5. The bound $\widehat{\sigma} \geq \widetilde{\sigma}$ can be derived as follows:

$$
\begin{aligned}
\widetilde{\sigma} & =\sum_{(i, j) \in E}\left(\overline{\mathcal{Q}}_{i j}-\overline{\mathcal{Q}}_{j i}\right)^{2} \frac{1}{\mathcal{Q}_{i j}+\mathcal{Q}_{j i}} \\
& \leq \sum_{(i, j) \in E}\left(\overline{\mathcal{Q}}_{i j}^{2}+\overline{\mathcal{Q}}_{j i}^{2}\right) \overline{\frac{1}{\mathcal{Q}_{i j}+\mathcal{Q}_{j i}}} \leq \sum_{(i, j) \in E} \overline{\mathcal{Q}}_{i j}^{2} \overline{\frac{1}{\mathcal{Q}_{i j}}}+\sum_{(i, j) \in E} \overline{\mathcal{Q}}_{j i}^{2} \overline{\frac{1}{\mathcal{Q}_{j i}}}=\widehat{\sigma} .
\end{aligned}
$$

\section{Appendix B. Periodic solutions of the Continuity equation}

In this short appendix we illustrate two possible approaches to find good perturbations $(m, R)$ in Section 5. We present just the general ideas since a complete development would be long and model-dependent.

B.1. Time dependent Schnakenberg theory. We consider a cycle

$$
C=\left(i_{1}, i_{2}, \ldots, i_{N}, i_{1}\right)
$$

of the transition graph $(V, E)$ and look for pairs $(R, m)$ satisfying properties $(\mathrm{P} 1)$, (P2), (P3) in Section 5, just restricted to this cycle. Since we have a one dimensional ring this is relatively easy. The continuity equation reduces to

$$
\dot{m}_{i_{k}}=R_{i_{k-1} i_{k}}-R_{i_{k} i_{k+1}}, \quad k=1, \ldots, N,
$$

where the sums $k \pm 1$ are modulo $N$. The general solution is therefore given by

$$
\left\{\begin{array}{l}
m_{i_{k}}(t)=M_{k}+\widehat{\alpha}_{k-1}(t)-\widehat{\alpha}_{k}(t) \\
R_{i_{k} i_{k+1}}(t)=\alpha_{k}(t)
\end{array}\right.
$$

where $\alpha_{k}, k=1,2, \ldots, N$, are arbitrary time-periodic functions such that $\int_{0}^{\tau} \alpha_{k}(t) d t$ does not depend on $k$. The functions $\widehat{\alpha}_{k}$ are the corresponding primitives of $\alpha_{k}$ and $M_{k}$ are arbitrary numbers such that $\sum_{k=1}^{N} M_{k}=0$.

A special degenerate case is obtained as follows. Consider two particles, performing time-periodic deterministic trajectories on the cycle $C$. Call $m$ the difference of the empirical densities associated to the trajectory of the first and of the second particle, respectively. Similarly call $R$ the difference of the empirical flows. Then the pair $(m, R)$ satisfies properties (P1), (P2), (P3).

Once obtained solutions on elementary cycles, a trial pair $(m, R)$ satisfying properties $(\mathrm{P} 1),(\mathrm{P} 2),(\mathrm{P} 3)$ for the transition graph $(V, E)$ can be obtained as a combination of them. The classic Schnakenberg theory allows to construct divergence free flows using cycles. This approach in a sense is a time-dependent version of this theory, giving solutions of the continuity equation using the cycle decomposition.

B.2. Perturbations from Markov models. Another possible approach that can be useful in specific situations is obtained by the following observation. Consider a Markov chain with periodic rates $\widetilde{w}$. If we call $\widetilde{\pi}$ its invariant time periodic distribution and $\widetilde{\mathcal{Q}}_{i j}=\widetilde{\pi}_{i}(t) \widetilde{w}_{i j}(t)$ the corresponding asymptotic flow we have that $\widetilde{\pi}$ and $\widetilde{\mathcal{Q}}$ are related by the continuity equation. We can therefore fix the pair $(m, R)$ by $m_{i}(t)=M_{i}-\widetilde{\pi}_{i}(t)$ and $R_{i j}(t)=\widetilde{\mathcal{Q}}_{i j}(t)$, where the arbitrary numbers $M_{i}$ satisfy the condition $\sum_{i} M_{i}=1$. This special way of proceeding can be useful in specific cases where there is a simple and natural periodic chain to be introduced. 
For both approaches we just discussed the constraints given by $(P 1),(P 2)$ and $(P 3)$ in Section 5. To really implement the methods it is necessary to satisfy also the additional constraint $(P 4)$ in Section 5. This further restriction has to be imposed on the perturbations discussed above.

\section{Appendix C. Derivation of Theorem 4}

We use the same notation introduced in Section 7. The GTUR (7.6) is an immediate consequence of (7.5) and the identity $2 D_{F}=1 / I_{F}^{\prime \prime}\left(y_{F}\right)$. We now explain how to derive (7.5) .

Recall that $\Theta_{\tau}$ is the family of piecewise constant paths $\Gamma:[0, \tau] \rightarrow V$. We denote by $\mathcal{P}\left(\Theta_{\tau}\right)$ the set of probability measures on $\Theta_{\tau}$. The expectation w.r.t. $P$ will be denoted by $E[\cdot]$.

We first focus on the empirical object

$$
Q^{(n)}:=\frac{1}{n} \sum_{j=0}^{n-1} \delta_{\left(X_{j \tau+s}\right)_{0 \leq s \leq \tau}} \in \mathcal{P}\left(\Theta_{\tau}\right) .
$$

Note that $Q^{(n)}$ is the empirical measure of the Markov chain $\left(W_{k}\right)_{k>0}$ on $\Theta_{\tau}$, where $W_{k}:=\left(X_{k \tau+s}\right)_{0 \leq s \leq \tau}$. We point out that in this Appendix $Q^{(n)}$ is defined as in (C.1) in order to make the notation closer to the one in [42, in particular $Q^{(n)}$ is not the empirical flow as in the rest of the file (cf. Section 2.15).

The link with the empirical functional (7.3) is given by the identity

$$
Y_{n}^{(F)}=\int_{\Theta_{\tau}} Q^{(n)}(d \Gamma) F(\Gamma) .
$$

To have $I(Q)<+\infty$ we need that

$$
Q\left(\Gamma_{0}=i\right)=Q\left(\Gamma_{\tau}=i\right) \quad \forall i \in V .
$$

This follows from the fact that $Q^{(n)}\left(\Gamma_{0}=i\right)=Q^{(n)}\left(\Gamma_{\tau}=i\right)+O(1 / n)$ (simply, the final value of $\left(X_{j \tau+s}\right)_{0 \leq s \leq \tau}$ equals the initial value of $\left.\left(X_{(j+1) \tau+s}\right)_{0 \leq s \leq \tau}\right)$.

As discussed in Subsection C.1 $Q^{(n)}$ fulfills an LDP with speed $n$ and the associated $\mathrm{LD}$ rate functional $I$ satisfies the inequality

$$
I(Q) \leq H(Q \mid P)
$$

for any $Q \in \mathcal{P}\left(\Theta_{\tau}\right)$ satisfying (C.3). By the contraction principle 25] we get that $Y_{n}^{(F)}$ satisfies an LDP with speed $n$, whose LD rate functional $I_{F}$ is given by

$$
I_{F}(y)=\inf \left\{I(Q): Q \in \mathcal{P}\left(\Theta_{\tau}\right), \int_{\Theta_{\tau}} Q(d \Gamma) F(\Gamma)=y\right\} .
$$

By combining (C.4) and (C.5) we have

$$
I_{F}(y) \leq H(Q \mid P) \quad \forall Q \in \mathcal{P}\left(\Theta_{\tau}\right) \text { fulfilling ([C.3) and } \int_{\Theta_{\tau}} Q(d \Gamma) F(\Gamma)=y \text {. }
$$

We apply (C.6) with some special $Q=Q^{y}$ that we take absolutely continuous w.r.t. $P$. Since $y_{F} \neq 0$, for some function $G$ we can write $Q^{y}$ as

$$
\frac{d Q^{y}}{d P}=1+\frac{y-y_{F}}{y_{F}}(1-G) \text {. }
$$


Due to (C.7), the properties $Q^{y} \in \mathcal{P}\left(\Theta_{\tau}\right), \int_{\Theta_{\tau}} Q^{y}(d \Gamma) F(\Gamma)=y$ and (C.3) are satisfied if and only if

$$
E[G]=1, \quad E[F G]=0 \quad \text { and } \quad E\left[G \mathbb{1}_{\Gamma_{0}=i}\right]=E\left[G \mathbb{1}_{\Gamma_{\tau}=i}\right] \forall i \in V .
$$

We claim that, using that $F \circ \mathcal{R}_{\tau}=-F$, the last two conditions on (C.8) are always satisfied if

$$
\frac{G}{G \circ \mathcal{R}_{\tau}}=\frac{d P \circ \mathcal{R}_{\tau}}{d P}
$$

where $P \circ \mathcal{R}_{\tau}$ is the probability on $\Theta_{\tau}$ defined as $P \circ \mathcal{R}_{\tau}(A):=P\left(\mathcal{R}_{\tau}(A)\right)$ for $A \subset \Theta_{\tau}$ measurable.

Let us derive the claim. Assuming (C.9), we can write

$$
\begin{aligned}
E[G F] & =-E\left[G\left(F \circ \mathcal{R}_{\tau}\right)\right]=-\int_{\Theta_{\tau}} P \circ \mathcal{R}_{\tau}(d \Gamma)\left(G \circ \mathcal{R}_{\tau}\right)(\Gamma) F(\Gamma) \\
& =-E\left[\frac{d P \circ \mathcal{R}_{\tau}}{d P}\left(G \circ \mathcal{R}_{\tau}\right) F\right]=-E[G F],
\end{aligned}
$$

thus implying that $E[G F]=0$ (note that (C.9) has been used to get the last identity). Similarly one can derive the last condition of (C.8) from (C.9).

One possible choice for (C.9) satisfying the constrain $E[G]=1$ is

$$
G=\frac{\left(1+e^{Z}\right)^{-1}}{E\left[\left(1+e^{Z}\right)^{-1}\right]}, \quad e^{-Z}=\frac{d P \circ \mathcal{R}_{\tau}}{d P} .
$$

Note that $E[Z]=\tau \sigma_{\text {naive }}($ cf. (7.1) and (7.2)).

Remark C.1. Let us naively think of the path space as countable. Writing $G_{\Gamma}$ for $G(\Gamma), \widetilde{\Gamma}:=\mathcal{R}_{\tau}(\Gamma)$ and setting $C_{\Gamma}:=P_{\Gamma} G_{\Gamma}$, (C.9) is equivalent to $C_{\Gamma}=C_{\widetilde{\Gamma}}$, while (C.7) reads

$$
Q_{\Gamma}^{y}=P_{\Gamma}+\frac{y-y_{F}}{y_{F}}\left(P_{\Gamma}-C_{\Gamma}\right) .
$$

The identity $E(G)=1$ would read $\sum_{\Gamma} C_{\Gamma}=1$. The choice $C_{\Gamma}=\frac{1}{\mathcal{N}} \frac{P_{\Gamma} P_{\widetilde{\Gamma}}}{P_{\Gamma}+P_{\widetilde{\Gamma}}}$ as in [42] $(\mathcal{N}$ being the normalization constant) would correspond to

$$
G_{\Gamma}=\frac{C_{\Gamma}}{P_{\Gamma}}=\frac{1}{\mathcal{N}} \frac{P_{\widetilde{\Gamma}}}{P_{\Gamma}+P_{\widetilde{\Gamma}}},
$$

which is equivalent to

$$
\frac{1}{G_{\Gamma}}=\operatorname{const}\left(1+\frac{d P}{d P \circ \mathcal{R}}\right)=\operatorname{const}\left(1+e^{Z}\right) .
$$

The above form of $G$ is exactly the choice (C.11).

From now on $G$ is as in (C.11). For simplicity we write

$$
G=\frac{1}{\mathcal{N}} \frac{1}{1+e^{Z}}, \quad \mathcal{N}=E\left[\left(1+e^{Z}\right)^{-1}\right] .
$$

By (C.6) we have

$$
I_{F}(y) \leq H\left(Q^{y} \mid P\right)=E\left[\frac{d Q^{y}}{d P} \ln \frac{d Q^{y}}{d P}\right]
$$


Using that $x \ln x=x-1+\frac{1}{2}(x-1)^{2}+o\left((x-1)^{2}\right)$, we obtain (recall that $E(G)=1$ )

$$
\begin{aligned}
I_{F}(y) & \leq \frac{1}{2} \frac{\left(y-y_{F}\right)^{2}}{y_{F}^{2}} E\left[(1-G)^{2}\right]+o\left(\left(y-y_{F}\right)^{2}\right) \\
& =\frac{1}{2} \frac{\left(y-y_{F}\right)^{2}}{y_{F}^{2}}\left(E\left[G^{2}\right]-1\right)+o\left(\left(y-y_{F}\right)^{2}\right) .
\end{aligned}
$$

Now observe that

$$
E\left[G^{2}\right]=\int P \circ \mathcal{R}_{\tau}(d \Gamma)\left(G \circ \mathcal{R}_{\tau}\right)^{2}=E\left[\frac{d P \circ \mathcal{R}_{\tau}}{d P}\left(G \circ \mathcal{R}_{\tau}\right)^{2}\right] .
$$

Using (C.9) we get that

$E\left[\frac{d P \circ \mathcal{R}_{\tau}}{d P}\left(G \circ \mathcal{R}_{\tau}\right)^{2}\right]=E\left[\frac{d P \circ \mathcal{R}_{\tau}}{d P} G^{2} \cdot\left(\frac{d P}{d P \circ \mathcal{R}_{\tau}}\right)^{2}\right]=E\left[G^{2} \frac{d P}{d P \circ \mathcal{R}_{\tau}}\right]=E\left[G^{2} e^{Z}\right]$.

As a byproduct of (C.16) and (C.17) we conclude that $E\left[G^{2}\right]=E\left[G^{2} e^{Z}\right]$ and therefore

$$
E\left[G^{2}\right]=\frac{1}{2} E\left[G^{2}\left(1+e^{Z}\right)\right]=\frac{1}{2 \mathcal{N}^{2}} E\left[\left(1+e^{Z}\right)^{-2}\left(1+e^{Z}\right)\right]=\frac{1}{2 \mathcal{N}}=\frac{1}{2 E\left[\left(1+e^{Z}\right)^{-1}\right]} .
$$

Inserting the above identity in (C.15) we get

$$
I_{F}(y) \leq \frac{1}{2} \frac{\left(y-y_{F}\right)^{2}}{y_{F}^{2}}\left(\frac{1}{2 E\left[\left(1+e^{Z}\right)^{-1}\right]}-1\right)+o\left(\left(y-y_{F}\right)^{2}\right) .
$$

We now claim that

$$
\frac{1}{\mathcal{N}}=\frac{1}{E\left[\left(1+e^{Z}\right)^{-1}\right]} \leq 1+e^{E[Z]}=1+e^{\tau \sigma_{\text {naive }}}
$$

(note that the identities in (C.20) follow from the definitions). By plugging (C.20) into (C.19) we get that

$$
I_{F}(y) \leq \frac{1}{4} \frac{\left(y-y_{F}\right)^{2}}{y_{F}^{2}}\left(e^{\tau \sigma_{\text {naive }}}-1\right)+o\left(\left(y-y_{F}\right)^{2}\right),
$$

which implies (7.5).

Inequality (C.20) corresponds to [42, Eq. (17)] and follows from a very tricky algebra in [42, App. A] that we adapt to our terminology. Since $E\left[e^{-Z}\right]=1$ (by the definition of $Z$ ), $P^{\prime}$ defined as $d P^{\prime}=\frac{1+e^{-Z}}{2} d P$ is a probability measure on $\Theta_{\tau}$. By applying Jensen's inequality w.r.t. this probability $P^{\prime}$ we have

$$
\ln \mathcal{N}=\ln E\left[\left(1+e^{Z}\right)^{-1}\right]=\ln E\left[\frac{1+e^{-Z}}{2} \frac{2 e^{-Z}}{\left(1+e^{-Z}\right)^{2}}\right] \geq E\left[\frac{1+e^{-Z}}{2} \ln \frac{2 e^{-Z}}{\left(1+e^{-Z}\right)^{2}}\right]
$$

Since $E\left[e^{-Z} Z\right]=-E[Z]$ (by the definition of $Z$ ), we have

$$
E[Z]=-E\left[\frac{e^{-Z}-1}{2} Z\right]
$$

Hence, setting $u:=e^{-Z}$, one gets a bound corresponding to [42, Eq. (A.1)]:

$$
\ln \mathcal{N}+E[Z] \geq E\left[\frac{1+u}{2} \ln \frac{2 u}{(1+u)^{2}}+\frac{u-1}{2} \ln u\right] .
$$


Since $\frac{1+a}{2} \ln \frac{2 a}{(1+a)^{2}}+\frac{a-1}{2} \ln a \geq(1-\ln 2) \frac{1+a}{2}-\frac{2 a}{a+1}$ for $a>0$ and since $d P^{\prime}=\frac{1+u}{2} d P$ is a probability, we can lower bound the r.h.s. of (C.22) by

$$
E\left[(1-\ln 2) \frac{1+u}{2}-\frac{2 u}{u+1}\right]=(1-\ln 2)-2 E\left[\frac{e^{-Z}}{e^{-Z}+1}\right]=(1-\ln 2)-2 \mathcal{N}
$$

Since $1-\ln 2-2 a \geq \ln (1-a)$ for all $a \geq 0$, one concludes from (C.22) and (C.23) that $\ln \mathcal{N}+E[Z] \geq \ln (1-\mathcal{N})$. This last estimate trivially implies (C.20).

C.1. Large deviations of $Q^{(n)}$. Given $Q \in \mathcal{P}\left(\Theta_{\tau}\right)$ we define, for $k, l \in V$,

$$
\left\{\begin{array}{l}
q_{k}=Q\left(\Gamma_{0}=k\right) \\
q_{k, l}=Q\left(\Gamma_{0}=k, \Gamma_{\tau}=\ell\right) .
\end{array}\right.
$$

We let $\bar{q}=\left(q_{k, l}\right)_{(k, l) \in V \times V}$. When we want to stress the dependence on $Q$, we write $q_{k}[Q], q_{k, l}[Q], \bar{q}[Q]$. Recall that $P$ is the law on $\Theta_{\tau}$ of the random trajectory $\left(X_{s}\right)_{0 \leq s \leq \tau}$ when $X_{0}$ has initial distribution $\pi_{0}$. We then set $p_{k, l}:=q_{k, l}[P]$ and $p_{k}:=q_{k}[P]$.

We consider the pair empirical measure

$$
\bar{q}^{(n)}:=\frac{1}{n} \sum_{j=0}^{n-1} \delta_{\left(X_{j \tau}, X_{(j+1) \tau}\right)},
$$

and observe that $\bar{q}^{(n)}:=\bar{q}\left[Q^{(n)}\right]$. By [25, Thm. IV.3], $\bar{q}^{(n)}$ satisfies a LD principle with speed $n$ and rate functional $I_{2}$ defined as follows. Let $\widetilde{\mathfrak{M}}_{1}(V \times V)$ be given by the families

$$
\bar{c}=\left(c_{k l}\right)_{(k, l) \in V \times V}
$$

with

$$
c_{k l} \geq 0, \quad \sum_{k} \sum_{l} c_{k, l}=1, \quad \sum_{k} c_{k l}=\sum_{k} c_{l k} .
$$

If $\bar{q} \in \widetilde{\mathfrak{M}}_{1}(V \times V)$, then

$$
I_{2}(\bar{q}):=\sum_{k, l} q_{k, l} \ln \frac{q_{k l}}{q_{k} P\left(X_{\tau}=l \mid X_{0}=k\right)}=\sum_{k, l} q_{k, l} \ln \frac{q_{k l}}{p_{k l}}-\sum_{q_{k}} q_{k} \ln \frac{q_{k}}{p_{k}},
$$

otherwise $I_{2}(\bar{q}):=+\infty$.

Proposition C.2. $Q^{(n)}$ satisfies a LDP with speed $n$ and rate function

$$
I(Q)=I_{2}(\bar{q})+\sum_{k, l} q_{k, l} H\left[Q_{k l} \mid P_{k l}\right] \quad \forall Q \in \mathcal{P}\left(\Theta_{\tau}\right),
$$

where

- $\bar{q}=\bar{q}[Q], q_{k, l}=q_{k, l}[Q]$;

- $I_{2}$ is the pair empirical measure LD functional for the discrete time homogeneous Markov chain $\left(X_{n \tau}\right)_{n \geq 0}$, which has invariant distribution $\pi_{0}$;

- $Q_{k, l}:=Q\left(\cdot \mid X_{0}=k, X_{\tau}=l\right)$;

- $P_{k, l}:=P\left(\cdot \mid X_{0}=k, X_{\tau}=l\right)$;

- $H\left[Q_{k l} \mid P_{k l}\right]$ is the relative entropy of the probability $Q_{k l}$ w.r.t. the probability $P_{k l}$. 
Proof. We only sketch the main idea which can be easily formalized. We will make some abuse of notation for the sake of intuition. Recall that $\bar{q}=\bar{q}[Q]$. Given $Q \in \mathcal{P}\left(\Theta_{\tau}\right)$ we have

$$
P\left(Q^{(n)}=Q\right)=P\left(Q^{(n)}=Q, \bar{q}^{(n)}=\bar{q}\right)=P\left(Q^{(n)}=Q, \mid \bar{q}^{(n)}=\bar{q}\right) P\left(\bar{q}^{(n)}=\bar{q}\right)
$$

By [25, Thm. IV.3] we have

$$
P\left(\bar{q}^{(n)}=\bar{q}\right)=e^{-n I_{2}(\bar{q})} .
$$

Consider the time interval $[0, n \tau]$ as the union $\cup_{j=0}^{n-1} A_{j}$, where $A_{j}=[j \tau,(j+1) \tau]$. If we know that $\bar{q}^{(n)}=\bar{q}$, then for each pair $(k, l)$ we know that there are $q_{k l} n$ intervals $A_{j}$ 's where the trajectory starts at $k$ and ends at $l$ (we call such a random set of intervals $\mathcal{A}_{k l}$ ). If we further condition on these intervals, then the random trajectories on $A_{j}$, with $A_{j} \in \mathcal{A}_{k l}$, behave as $n q_{k l}$ i.i.d. random variables with value in $\Theta_{\tau}$ and with distribution $P_{k, l}$. Moreover, the random objects involved are independent when varying $(k, l)$. By applying Cramér's Theorem and the independence we conclude that

$$
P\left(Q^{(n)}=Q, \mid \bar{q}^{(n)}=\bar{q}\right)=\prod_{(k, l)} e^{-q_{k l} n H\left[Q_{k, l} \mid P_{k, l}\right]} .
$$

The thesis then follows as a byproduct of (C.27), (C.28) and (C.29).

Note that, since in (C.26), $\bar{q}=\bar{q}[Q]$ and $Q \in \mathcal{P}\left(\Theta_{\tau}\right)$, we get that $\bar{q} \in \widetilde{\mathfrak{M}}_{1}(V \times V)$ if and only if $\sum_{k} q_{k l}=\sum_{k} q_{l k}$ for each $l \in V$, which is equivalent to (C.3). As a consequence, if $Q \in \mathcal{P}\left(\Theta_{\tau}\right)$ fulfills (C.3) then

$$
I_{2}(\bar{q})=\sum_{k, l} q_{k, l} \ln \frac{q_{k l}}{p_{k l}}-\sum_{q_{k}} q_{k} \ln \frac{q_{k}}{p_{k}} \text { where } \bar{q}=\bar{q}[Q] .
$$

By combining (C.26) and (C.30) one easily gets that the LD rate functional $I(Q)$, for $Q \in \mathcal{P}\left(\Theta_{\tau}\right)$ fulfilling (C.3), can be written as

$$
I(Q)=H(Q \mid P)-\sum_{k} q_{k} \ln \frac{q_{k}}{p_{k}} .
$$

We derive (C.31) for completeness. Given $k, l \in V$ we set $\Theta_{\tau}(k, l):=\left\{\Gamma \in \Theta_{\tau}\right.$ : $\left.\Gamma_{0}=k, \Gamma_{\tau}=l\right\}$. Then, when $Q_{k, l} \ll P_{k, l}$ (the case $Q_{k, l} \ll P_{k, l}$ can be treated easily)

$$
\begin{aligned}
H\left[Q_{k, l} \mid P_{k, l}\right] & =\int_{\Theta_{\tau}(k, l)} Q_{k, l}(d \Gamma) \ln \frac{d Q_{k, l}}{d P_{k, l}}(\Gamma) \\
& =\frac{1}{q_{k, l}} \int_{\Theta_{\tau}(k, l)} Q(d \Gamma) \ln \frac{d Q}{d P}(\Gamma)-\ln \frac{q_{k, l}}{p_{k, l}} .
\end{aligned}
$$

By combining the above equation with (C.25) and (C.26) we get the (C.31).

As a consequence we have

$$
I(Q) \leq H(Q \mid P)
$$

for any $Q \in \mathcal{P}\left(\Theta_{\tau}\right)$ fulfilling (C.3).

Acknowledgements. A.F. and D.G. thank the Laboratoire J.A Dieudonné in Nice for the kind hospitality and the University of Nice for the financial support. 


\section{REFERENCES}

[1] A.C. Barato, R. Chetrite, A. Faggionato, D. Gabrielli; Bounds on current fluctuations in periodically driven systems. New J. Phys. 20103023 (2018).

[2] A.C. Barato, U. Seifert; Universal Bound on the Fano Factor in Enzyme Kinetics. J. Phys. Chem. B 119 6555-6561 (2015).

[3] A.C. Barato, U. Seifert; Thermodynamic uncertainty relation for biomolecular processes. Phys. Rev. Lett. 114, 158101 (2015).

[4] A.C. Barato, U. Seifert; Cost and precision of Brownian clocks. Phys. Rev. X 6041053 (2016).

[5] L. Bertini, R. Chetrite, A. Faggionato, D. Gabrielli; Level 2.5 large deviations for continuoustime Markov chains with time periodic rates. Ann. Henri Poincaré, 19, 31973238 (2018).

[6] L. Bertini, A. Faggionato, D. Gabrielli; Large deviations of the empirical flow for continuous time Markov chains. Ann. Inst. H. Poincaré Probab. Statist. 51, 867-900 (2015).

[7] L. Bertini, A. Faggionato, D. Gabrielli; Flows, currents, and cycles for Markov chains: Large deviation asymptotics. Stoch. Proc. Appl. 125, 2786-2819 (2015).

[8] G. Bisker, M. Polettini, T.R. Gingrich, J.M. Horowitz; Hierarchical bounds on entropy production inferred from partial information. J. Stat. Mech.: Theor. Exp. (2017) 093210

[9] K. Brandner, T. Hanazato, K. Saito; Thermodynamic Bounds on Precision in Ballistic Multiterminal Transport. Phys. Rev. Lett. 120, 090601 (2018)

[10] D. Chiuchiù, S. Pigolotti; Mapping of uncertainty relations between continuous and discrete time. Phys. Rev. E 97, 032109 (2018)

[11] A. Dechant; Multidimensional thermodynamic uncertainty relations. arXiv:1809.10414 (2018)

[12] A. Dechant, S. Sasa; Current fluctuations and transport efficiency for general Langevin systems. J. Stat. Mech. (2018) 063209

[13] A. Dechant, S. Sasa; Entropic bounds on currents in Langevin systems. Phys. Rev. E 97, 062101 (2018)

[14] A. Dembo, O. Zeitouni; Large deviations techniques and applications. Second edition. SpringerVerlag, New York, 1998.

[15] J.-D. Deuschel, D.W. Stroock; Large deviations. Academic Press, San Diego, 1989.

[16] I. Di Terlizzi, M. Baiesi; Kinetic uncertainty relation. arXiv:1809.06410 (2018)

[17] S. Erbas-Cakmak, D.A. Leigh, C.T. McTernan, A.L. Nussbaumer; Artificial molecular machines. Chem. Rev. 115, 10081-10206 (2015)

[18] A. Faggionato, D. Gabrielli, Ribezzi Crivellari M.; Non-equilibrium thermodynamics of piecewise deterministic Markov processes. J. Stat. Phys. 137, 259-304 (2009).

[19] J.P. Garrahan; Simple bounds on fluctuations and uncertainty relations for first-passage times of counting observables. Phys. Rev. E 95, 032134 (2017).

[20] T.R. Gingrich, J. M. Horowitz; Fundamental Bounds on First Passage Time Fluctuations for Currents. Phys. Rev. Lett. 119, 170601 (2017).

[21] T.R. Gingrich, J.M. Horowitz, N. Perunov, J.L. England; Dissipation Bounds All Steady-State Current Fluctuations. Phys. Rev. Lett. 116, 120601 (2016).

[22] T.R. Gingrich, G.M. Rotskoff, J.M. Horowitz; Inferring dissipation from current fluctuations. J. Phys. A: Math. Theor. 50, 184004 (2017).

[23] J. Guioth, D. Lacoste; Thermodynamic bounds on equilibrium fluctuations of a global or local order parameter. Europhysics Letters, 115, 6, 60007, (2016).

[24] C. Hyeon, W. Hwang; Physical insight into the thermodynamic uncertainty relation using Brownian motion in tilted periodic potentials. Phys. Rev. E 96, 012156, (2017).

[25] F. den Hollander; Large deviations. Fields Institute Monographs, AMS, Providence RI, 2000.

[26] J.M. Horowitz, T.R. Gingrich; Proof of the finite-time thermodynamic uncertainty relation for steady-state currents. Phys. Rev. E 96, 020103(R) (2017).

[27] T. Koyuk, U. Seifert, P. Pietzonka; A generalization of the thermodynamic uncertainty relation to periodically driven systems. Preprint, arXiv:1809.02113 (2018).

[28] K. Macieszczak, K. Brandner, J.P. Garrahan; Unified Thermodynamic Uncertainty Relations in Linear Response. Phys. Rev. Lett. 121,130601 (2018)

[29] C. Maes; Frenetic Bounds on the Entropy Production. Phys. Rev. Lett. 119, 160601, (2017)

[30] C. Maes, K. Netočný; Canonical structure of dynamical fluctuations in mesoscopic nonequilibrium steady states. Europhys. Lett. 82, 30003 (2008). 
[31] I.A. Martínez, É. Roldán, L. Dinis, R.A. Rica; Colloidal heat engines: a review. Soft Matter 13, 22-36 (2017).

[32] C. Nardini, H. Touchette; Process interpretation of current entropic bounds. Eur. Phys. J. B 91, 1434 (2018).

[33] M. Nguyen, S. Vaikuntanathan; Design principles for nonequilibrium self-assembly. Proc. Natl. Acad. Sci. 113, 14231-14236 (2016).

[34] P.T. Nyawo, H. Touchette; Large deviations of the current for driven periodic diffusions. Phys. Rev. E 94032101 (2016).

[35] P. Pietzonka, A.C. Barato, U. Seifert; Universal bound on current fluctuations. Phys. Rev. E 93, $052145(2016)$

[36] P. Pietzonka, A.C. Barato, U. Seifert; Universal bound on the efficiency of molecular motors. J. Stat. Mech. 124004 (2016).

[37] P. Pietzonka, A.C. Barato, U. Seifert; Affinity- and topology-dependent bound on current fluctuations. J. Phys. A 49 34LT01 (2016)

[38] P. Pietzonka, F. Ritort, U. Seifert; Finite-time generalization of the thermodynamic uncertainty relation. Phys. Rev. E 96, 012101 (2017).

[39] P. Pietzonka, U. Seifert; Universal trade-off between power, efficiency, and constancy in steadystate heat engines. Phys. Rev. Lett. 120, 190602 (2018).

[40] S. Pigolotti, I. Neri, É. Roldán, F. Jülicher; Generic Properties of Stochastic Entropy Production. Phys. Rev. Lett. 119, 140604, (2017).

[41] M. Polettini,A. Lazarescu, M. Esposito; Tightening the uncertainty principle for stochastic currents. Phys. Rev. E 94, 052104 (2016).

[42] K. Proesmans, C. Van den Broeck; Discrete-time thermodynamic uncertainty relations, Europhysics Letters 119, 20001 (2017).

[43] J. Schnakenberg; Network theory of microscopic and macroscopic behavior of master equation systems. Rev. Mod. Phys. 48, 571-585 (1976).

[44] U. Seifert; Stochastic thermodynamics, fluctuation theorems and molecular machines. Rep. Prog. Phys. 75126001 (2012).

[45] K. Sekimoto; Stochastic energetics. Lecture Notes in Physics 799, Springer Verlag, Berlin, 2010.

[46] H. Touchette; The large deviation approach to statistical mechanics. Phys. Rep. 478, 1-69 (2009)

Andre Cardoso Barato

Department of Physics, University of Houston, Houston, Texas 77204, USA

E-mail address: barato@uh.edu

Raphael Chetrite

CNRS, Laboratoire J.A. Dieudonné, Université Côte D'Azur

06108 Nice Cedex 2, France.

E-mail address: rchetrit@unice.fr

Alessandra Faggionato

Dipartimento di Matematica, Università di Roma 'La Sapienza'

P.Le Aldo Moro 2, 00185 Roma, Italy

E-mail address: faggiona@mat.uniroma1.it

DAVIDE GabRielli

DISIM, UNIVERSITÀ DELL'AQUILA

Via Vetoio, Loc. Coppito, 67100 L'Aquila, Italy

E-mail address: gabriell@univaq.it 